\title{
Internal insulation condition identification for high-voltage capacitor voltage transformers based on possibilistic fuzzy clustering
}

\author{
A. Zhan Meng, ${ }^{1}$ B. Hongbin Li, ${ }^{1}$ C. Qing Chen, ${ }^{1}$ a) and D. Chan Hwang See ${ }^{2}$ \\ ${ }^{1)}$ School of Electrical and Electronic Engineering, Huazhong University of Science and Technology, Wuhan 430074, \\ China \\ ${ }^{2)}$ School of Engineering and the Built Environment, Edinburgh Napier University, Edinburgh, \\ $U K$
}

(Dated: 16 December 2019)

\begin{abstract}
The internal insulation condition of capacitor voltage transformers (CVTs) is a key influence factor to its measurement performance and safe operation, but the internal insulation would be aged along with long-time operation and degraded influenced by environmental factors, once the insulation degradation grows, serious damages and even explosion may happen to CVTs, so it is necessary to monitor the internal insulation condition of CVTs, and the fault type and fault degree need to be identified. In this paper, a data-driven internal insulation condition identification method for CVTs is proposed. Both the amplitude and phase of the output voltage of CVTs are collected, then recognition models based on the combination of the output voltages and distribution topology of CVTs in substations are built, a possibilistic fuzzy clustering method is used to monitor the internal insulation condition of CVTs, different type and different degree of insulation faults could be identified effectively. Finally, the proposed method is verified by several cases, not only the preset typical faults in the method can be identified effectively, but also the faults beyond the preset faults could be diagnosed.
\end{abstract}

\section{INTRODUCTION}

The capacitor voltage transformers (CVTs) have already been the most widely used voltage transformer in the high voltage (HV) and ultra-high voltage (UHV) power system ${ }^{1-3}$. The internal structure of CVTs is much more complicated compared with the conventional electromagnetic potential transformers (PTs), which leads to worse long-term stability and susceptibility to external electromagnetic interference 4,5 . Besides, the insulation of CVTs is prone to be aged after long-term operation, and the over voltage in the power system and the partial discharge in the CVT may cause irreversible damage to the insulation of CVTs ${ }^{6}$. A CVT whose insulation is faulted cannot convert the primary voltage into secondary voltage correctly, even worse, explosion may occur in CVTs, which is a threat to the safety of the power system. Therefore, it is necessary to monitor the insulation condition of CVTs, and the fault type of insulation needs to be diagnosed in time.

At present, the most common methods to obtain the insulation state of CVTs are conventional electric bridge detection and capacitive current monitoring. The electric bridge method needs the monitored CVTs out of operation ${ }^{7,8}$ and the capacitive current monitoring method needs extra acquisition device to collect the output voltage and leakage current of CVTs synchronously ${ }^{9}$, then the capacitance and dielectric loss of CVTs can be derived approximatively. However, the leakage current is less than $1 \mathrm{~mA}$ generally, so it is hard to guarantee its acquisition precision. In recent years, some insulation condition monitoring methods with brand-new concepts have been proposed. Li proposed a dielectric loss factor measurement approach based on sampling sequence reconstruction for capacitive equipment ${ }^{10}$, this method also needs to monitoring

a)Electronic mail: wfr101@163.com the voltage and current synchronously, and it is not particular to CVTs, the specific type of insulation faults cannot be diagnosed. Daryani proposed an evidence theory-based insulation aging identification approach for CVTs ${ }^{11}$, the output voltage, the negative sequence components, the zero sequence components and the park transformation parameter of groups of CVTs are considered to generate the indicators of insulation condition, but the case studies illustrate that this method could only detect more than three capacitors outage, the dielectric loss is not considered in it. Besides, the influence of the actual fluctuations of power system is not discussed either.

This paper proposed a data-driven internal insulation conditon monitoring method for CVTs. This method is based on the possibilistic fuzzy c-means clustering algorithm (PFCM), and it combines the physical model of the insulation structure and the output voltage datasets of groups of CVTs, the insulation condition of CVTs and the possibility of some typical faults occurrence are given by applying the PFCM to the output voltage datasets. The rest of this paper is organised as follows. Section II points out the main reason for the internal insulation degradation of CVTs, and an equivalent model to calculate the additional errors caused by the internal insulation faults is proposed. Section III introduces the PFCM firstly, then the specific insulation fault identification method is detailed. Section IV presents some cases, and conclusions are drawn in Section V finally.

\section{INTERNAL INSULATION OF CVTS}

As is shown in Fig. 1, A CVT mainly consists of a capacitive voltage divider, a compensation reactor, an intermediate transformer and a damper ${ }^{12}$. The capacitive voltage divider converts the primary voltage into the medium voltage, which is the input voltage of the intermediate transformer. The secondary voltage of the intermediate transformer is the output 
voltage of the CVT. The capacitive voltage divider consists of hundreds of same capacitor elements in series, and all the capacitors are immersed in the insulating oil in an insulating porcelain bushing. The intermediate transformer is a conventional PT with low primary voltage, and it is installed in the sealed tank with the compensation reactor and the damper, it is difficult for its low primary voltage to cause an interturn short circuit, which means its insulation is reliable and stable.

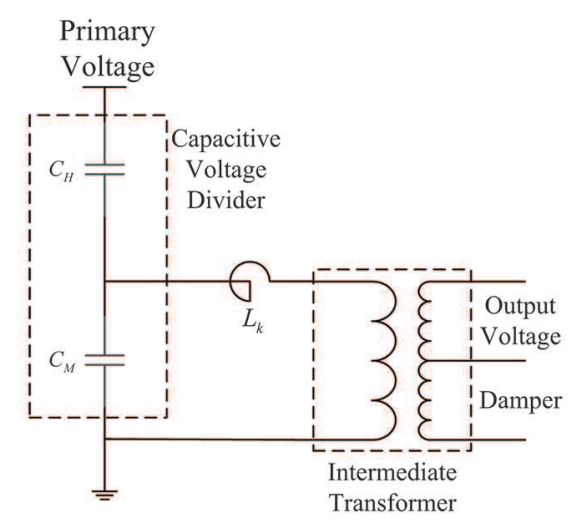

FIG. 1. Basic structure of CVTs

\section{A. CVTs internal insulation degradation analysis}

For the capacitive voltage divider, the insulation can be divided into external insulation and internal insulation. The external insulation fault is mainly due to the leakage current caused by the contamination on the surface of insulation bushing. The leakage current on the outer surface of the porcelain bushing would flows into the voltage divider at the connections between bushings, which have influence on the medium voltage directly. Although the contamination on the bushing would increase the leakage current, the ratio error variance of CVTs is still lower than $0.1 \%$ even if the leakage current is more than $25 \mathrm{~mA}^{13}$, which is corresponding to extremely heavy pollution, so the external surface contamination is not the main influence factor to the measurement performance of CVTs. Therefore, the potentially troublesome internal insulation is mainly the insulation of the capacitive voltage divider, which is susceptible to various influence factors, such as temperature, interior moisture, overvoltage, partial discharge, aging, etc. The detailed analysis is as follows.

1. Temperature: The capacitors in the capacitive voltage divider contain polypropylene (PP) film medium, both the capacitance and the dielectric loss of these capacitors are influenced by the temperature just like other common capacitors. The capacitance of these capacitors decreases as the temperature increases because of the negative temperature coefficient of PP film ${ }^{14}$. As for the dielectric loss of the PP film capacitor, it mainly consists of polarization loss and conduction loss, the conduction loss always increases with the increase in temperature, while the polarization loss increases to the maximum first and then decreases. The polarization loss dominates the dielectric loss of capacitor when the temperature is relatively low, so the variation trend of the dielectric loss is consistent with the polarization loss at relatively low temperature. But the conduction loss would increases sharply at higher temperature, then the dielectric loss of capacitors would increases as the higher temperature increases as result.

2. Aging: There is no moisture in the normal capacitive voltage divider, but the aged external insulation cannot prevent the intrusion of the moisture, thus the dielectric loss of the capacitors would be larger than the normal. Besides, as the moisture turns into tiny bubbles in capacitors with heat, then the partial discharge would happen in the damp capacitors, which would results in breakdown easily ${ }^{15}$.

3. Partial discharge: Each capacitor in the voltage divider is winded with two aluminum film electrodes and multilayer PP film medium generally. There would be bubbles or air gaps in the capacitors once some abnormalities happen during the manufacture, then partial discharge would likely happen at these weakness points ${ }^{16}$. The partial discharge would deteriorate gradually and leads to capacitor breakdown finally, which means the measurement performance of CVT is heavily effected.

4. Overvoltage: CVTs are connected to the HV bus directly, the overvoltage in the power system would get in the capacitive voltage divider directly, then the first few HV capacitors are most likely be damaged. Besides, CVTs contain capacitors and inductive elements, the inductive reactance is slightly larger than the capacitive reactance to avoid the internal resonance, but the instantaneous process in the power system may lead to saturation to the inductive elements ${ }^{17}$, which means the medium voltage capacitors in CVTs would be breakdown by the internal resonance overvoltage.

All the internal insulation faults would result in the variance of the dielectric loss and the capacitance, as a consequence, the proportion of the reactance of the HV capacitors and the MV capacitors deviate its their nominal values, which means the transformation ratio of the capacitive voltage divider would deviate from its nominal value.

The output voltage of CVT is the product of the primary voltage, the transformation ratio of the capacitive voltage divider and the transformation ratio of the intermediate transformer. Therefore, the faults of internal insulation would impact the output voltage of CVTs directly, that is the faults of internal insulation would result in additional ratio error and phase error to CVTs.

\section{B. Equivalent model of CVTs}

The abnormal internal insulation mainly results in capacitor breakdown and excessive dielectric loss. Generally, a 
nonideal capacitor can be equivalent to a capacitor in parallel with a resistor, and a T-type equivalent circuit could represents the intermedia transformer. Therefore, the equivalent circuit model which can embody the internal insulation condition of CVTs is shown in Fig. 2.

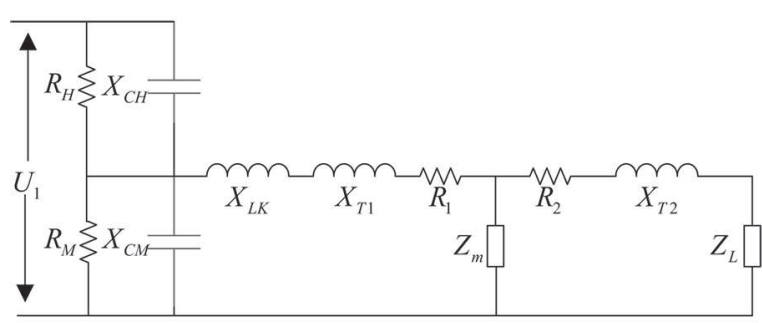

FIG. 2. Equivalent model of CVTs

In Fig. 2, $X_{C H}$ and $X_{C M}$ are equivalent reactance of $\mathrm{HV}$ capacitors and MV capacitors, $R_{H}$ and $R_{M}$ are equivalent resistance of the dielectric loss of the HV capacitors and MV capacitors respectively, $X_{T 1}, X_{T 2}, R_{1}, R_{2}$ and $Z_{m}$ are equivalent parameters of the intermedia transformer, $Z_{L}$ represents the secondary load. The rated equivalent reactance $X_{C H}, X_{C M}$, $X_{L K}$ and the rated dielectric loss are offered by the manufacturer, the equivalent parameters of the intermedia transformer can be obtained by the short-circuit test and the no-load test, and the equivalent secondary load is determined by the actual load of the operating CVTs.

Under normal conditions, the tangent dielectric loss of the capacitors should be less than $0.1 \%{ }^{18}$, then the equivalent resistance $R_{H}$ and $R_{M}$ approach positive infinity. If the dielectric loss of the capacitors exceed the limit, the corresponding equivalent resistance would be less than the normal. The equivalent resistance can be obtained as:

$$
R=\frac{1}{\omega C \tan \delta}
$$

Where $\omega$ is the angular frequency of the power system, $C$ is the corresponding capacitance, and $\tan \delta$ represents the dielectric loss. Besides, all the capacitors in a CVT are totally same, and the number of $\mathrm{HV}$ capacitor $n_{H}$, the number of MV capacitor $n_{M}$, the equivalent capacitance of HVcapacitor $C_{H}$ and the equivalent capacitance of MV capacitor $C_{M}$ should also be offered by the manufacturer, so that if $n$ capacitors are breakdown, the actual capacitance of $\mathrm{HV}$ capacitor and MV capacitor would change to:

$$
\begin{gathered}
C_{H}^{\prime}=\frac{n_{H}}{n_{H}-n} \cdot C_{H} \\
C_{M}^{\prime}=\frac{n_{M}}{n_{M}-n} \cdot C_{M}
\end{gathered}
$$

The additional ratio error and phase error caused by internal insulation faults can be obtained by simulating the output voltage of the model in Fig. 2 in normal condition and faulted condition respectively.

\section{METHOD}

As the analyses in Section II, the main symptoms of the internal insulation faults of CVTs are the additional ratio error and phase error, different fault degrees and fault types result in different additional errors. In this paper, the PFCM algorithm is introduced to recognize the internal insulation faults of CVTs, and recognition models based on the combination of the output voltages and the distribution topology of CVTs are proposed.

\section{A. Overview of PFCM}

The fuzzy c-means (FCM) algorithm is the most primitive fuzzy clustering algorithm, and it was proposed in the doctoral dissertation of J. Bezdek in $1973^{19}$. FCM generates fuzzy partitions and prototypes for any set of numerical data through iterative optimizing the objective function, and "membership" is generated to suggest which cluster the unexplored data belongs to ${ }^{20}$. FCM uses the probabilistic constraint, thus the memberships of a data point across substructures must sum to 1 , which results in the membership of a data point is not zero even if the outlier doesn't belong to any known cluster. To overcome the problem of FCM, possibilistic c-means (PCM) algorithm was proposed in $1993^{21}$. PCM generates "typicality" instead of "membership" as the degrees of possibility of data points belonging to clusters, it looses the constraint of FCM, so that all the typicalities of each data point can be any number between 0 and 1 , but PCM is very sensitive to initializations and it sometimes generates coincident clusters. To overcome the deficiencies and combine the superiorities of FCM and PCM, a possibilistic fuzzy c-means clustering algorithm (PFCM) was innovative proposed in $2005^{22}$.

\section{Basic definition}

The PFCM obtains the optimal classification of a dataset $X$ through iterative optimizing the objective function (4), which combines the objective functions of FCM and PCM.

$$
\begin{array}{r}
J_{m, \eta}(U, T, V ; X)=\sum_{k=1}^{n} \sum_{i=1}^{c}\left(a u_{i k}^{m}+b t_{i k}^{\eta}\right) \times\left\|x_{k}-v_{i}\right\|^{2} \\
+\sum_{i=1}^{c} \gamma_{i} \sum_{k=1}^{n}\left(1-t_{i k}\right)^{\eta}
\end{array}
$$

$u_{i k}$ has the same meaning as the membership of FCM, so it also subjects to the constraints $\sum_{i=1}^{c} u_{i k}=1$ and $u_{i k} \geq 0$. Similarly, $t_{i k}$ also has the same meaning as the typicality of PCM, and it only subjects to the constraint $0 \leq t_{i k} \leq 1 . a$ and $b$ define the relative importance of membership and typicality in the objective function, if $a=0$, (4) reduces to the the objective function of PCM, similarly, if $b=0$ and $\gamma=0$, (4) reduces to FCM. The reference [21] concluded that $b$ should be larger than $a$ so that the PFCM could reduces the influence of outliers like PCM, but $b$ should not be much larger than $a$, otherwise 
the effects of memberships will be eliminated. $c$ is the preset number of clusters in dataset $X . v_{i}$ is the center vector of the $\mathrm{i}$-th cluster, and $\left\|x_{k}-v_{i}\right\|$ represents the distance between $x_{k}$ and the $\mathrm{i}$-th cluster center (Euclidean distance is used in this paper). $m$ and $\eta$ are the weighting exponents, they control the relative weights placed on each distance vector, usually it takes $m=\eta=2$, and the penalty factor $\gamma_{i}$ could be calculated as:

$$
\gamma_{i}=K \cdot \frac{\sum_{k=1}^{n}\left(a u_{i k}^{m}+b t_{i k}^{\eta}\right) d_{i k}^{2}}{\sum_{k=1}^{n}\left(a u_{i k}^{m}+b t_{i k}^{\eta}\right)}
$$

where $d_{i k}=\left\|x_{k}-v_{i}\right\|$, and $K=1$ commonly.

The objective function could be minimized only if:

$$
\begin{gathered}
u_{i k}=\frac{1}{\sum_{j=1}^{c}\left(\frac{d_{i k}}{d_{j k}}\right)^{2 /(m-1)}} \\
t_{i k}=\frac{1}{1+\left(\frac{b}{\gamma_{i}} d_{i k}^{2}\right)^{1 /(\eta-1)}} \\
v_{i}=\frac{\sum_{k=1}^{n}\left(a u_{i k}^{m}+b t_{i k}^{\eta}\right) x_{k}}{\sum_{k=1}^{n}\left(a u_{i k}^{m}+b t_{i k}^{\eta}\right)}
\end{gathered}
$$

The weighting exponent $m$ and $\eta$ should be set before the iterative optimization of the objective function, besides, the initial cluster center $v_{o}$, the initial membership and typicality need to be randomly initialized. Then $\gamma, u, t, v$ will be updated according to $(5) \sim(8)$ until $\left\|v^{(l+1)}-v^{(l)}\right\|<\varepsilon$ (l is the number of iteration, $\varepsilon$ is the preset stop threshold) or $l>L$ ( $L$ is the preset maximum iterations), $v^{(l+1)}$ is the optimal cluster center vector, then the clustering by PFCM is completed.

\section{Fault indicators of PFCM}

Applying the PFCM to fault identification, the process consists of two parts, off-line modeling and on-line monitoring. The historical dataset is classified firstly, the dataset should contain the normal operating data and as many types of typical fault data as possible, then PFCM generates the typical clusters from the dataset. The memberships $u_{i k}$ and typicalities $t_{i k}$ of the new on-line monitoring dataset are calculated to represent the possibility of the typical faults occurence, the subscript $i$ represents which typical cluster $u_{i k}$ and $t_{i k}$ are relative to.

As discussed above, the membership $u_{i k}$ subjects to the constraints $\sum_{i=1}^{c} u_{i k}=1$ and $u_{i k} \geq 0$ as that of FCM, which means $u_{i k}$ are not closed to zero when a new kind of fault beyond the preset typical faults appears in the monitoring dataset, in other words, $u_{i k}$ cannot recognise faults beyond the typical faults, what's worse, the constrained $u_{i k}$ may leads misjudgement to the new faults. In this case, the typicality $t_{i k}$ is complementary to $u_{i k}, t_{i k}$ represents the possibility of the monitoring dataset belongs to each typical cluster, and it only subjects to $0 \leq t_{i k} \leq 1$. So all the $t_{i k}$ would be less than an optimal value and closed to zero once a new kind of fault appears in the monitoring dataset, the specific value of the threshold is determined by $b$ and $\eta$ as (7).

The specific fault identification procedure with the features of PFCM is shown in Fig. 3. $u_{0 k}$ and $t_{0 k}$ of the monitoring dataset are relative to the normal operation condition, which should be close to the optimal values, if any faults occur to the monitored CVT, $u_{0 k}$ and $t_{0 k}$ would drop down from the optimal value, which means the monitored CVT is out of the normal operation condition. According to the definitions of typicality $t_{i k}$ and membership $u_{i k}$, if all the typicalities of the monitoring data points is less than the optimal value significantly, it could by judged as an unknown fault happens, and all the $u_{i k}$ would be $\frac{1}{c}$ at the same time ( $c$ is the number of the preset typical fault type). Otherwise there must be some known faults happens to the monitored CVT and the membership $u_{m k}$ would be greater than all the other memberships, which means the monitored CVT is operating in the m-th typical fault condition.

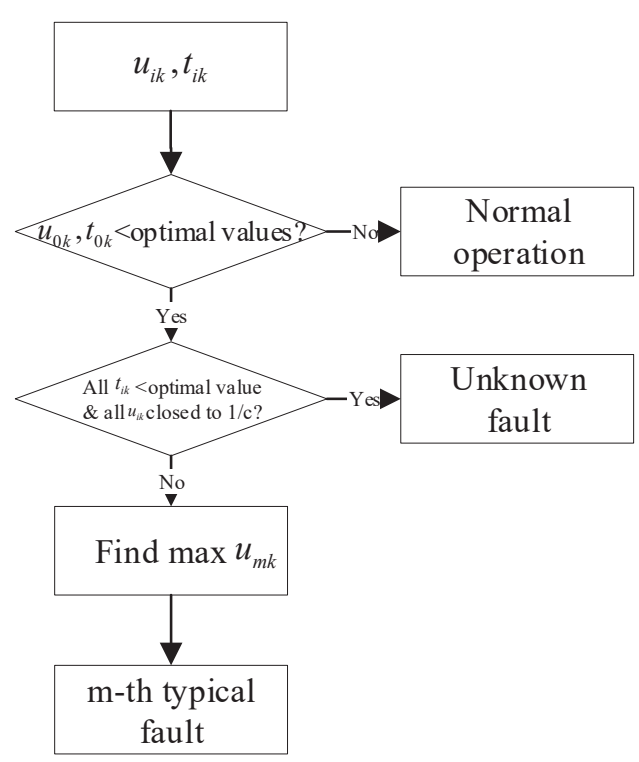

FIG. 3. Fault identification procedure of PFCM

\section{B. Insulation faults identification method}

As we know, the amplitude and phase of the secondary output voltage of CVTs are not stable variables, they fluctuate as the power grid fluctuation. Zhang established a recognition model to monitor the measurement error drift based on the three-phase voltage unbalance factor (VUF) ${ }^{23}$, however the VUF varied from $0.2 \%$ to $1.0 \%$ as the loads fluctuates ${ }^{24}$, which means VUF is not a reliable parameter for monitoring the error drift, let alone for faults identification. In this paper, recognition models based on the topology of groups of CVTs are proposed, the model is used to identify the internal insulation faults of CVTs along with PFCM. 


\section{Recognition model}

The actual transformation ratio of CVTs mainly comprises the nominal transformation ratio and its measurement error, then the amplitude and phase relationships between the primary voltage and the output voltage of the i-th CVT are:

$$
\begin{gathered}
V_{o i}=\left(1+\varepsilon_{i}\right) \cdot \frac{V_{p i}}{k_{r}} \\
\varphi_{o i}=\varphi_{p i}+\rho_{i}
\end{gathered}
$$

where $V_{p i}$ and $V_{o i}$ represent the amplitude of the primary voltage and output voltage voltage of the i-th CVT respectively, $\varepsilon_{i}$ is the ratio error and the $k_{r}$ is the rated transformation ratio of the CVT. Similarly, $\varphi_{p i}$ and $\varphi_{o i}$ are the phase of the primary voltage and the output voltage of the i-th CVT respectively, and $\rho_{i}$ is its phase error.

In the HV substations, it is common that multiple identical CVTs be connected to the incoming and outgoing transmission lines of same voltage level and same phase, which means the primary voltage of these CVTs are totally same, then their secondary output voltages contains same fluctuations as the bus voltage. Besides, it is generally known that CVT is susceptible to the electric field interference in the HV substation, the actual effect of the interference has been quantified by experiments in the Reference [5], the additional ratio error caused by electric interference is lower than $0.02 \%$ during operation, while the additional phase error is lower than $1^{\prime}$. Both the additional ratio error and phase error are far below the measurement tolerance of CVTs of 0.2 accuracy class, which means the effects of the electric interference are negligible compared with the measurement error.

Since all the $V_{p i}$ and $\varphi_{p i}$ are equal to these of the bus voltage, and all the CVTs of same voltage level have same transformation ratio, the relationship among output voltages of these CVTs can be expressed as (11) and (12).

$$
\begin{gathered}
\frac{V_{o 1}}{V_{o 2}}=\frac{1+\varepsilon_{1}}{1+\varepsilon_{2}} \\
\frac{V_{o 2}}{V_{o 3}}=\frac{1+\varepsilon_{2}}{1+\varepsilon_{3}} \\
\cdots \\
\frac{V_{o(i-1)}}{V_{o i}}=\frac{1+\varepsilon_{(i-1)}}{1+\varepsilon_{i}} \\
\frac{V_{o i}}{V_{o 1}}=\frac{1+\varepsilon_{i}}{1+\varepsilon_{1}} \\
\varphi_{o 1}-\varphi_{o 2}=\rho_{1}-\rho_{2} \\
\varphi_{o 2}-\varphi_{o 3}=\rho_{2}-\rho_{3} \\
\cdots \\
\varphi_{o(i-1)}-\varphi_{o i}=\rho_{(i-1)}-\rho_{i} \\
\varphi_{o i}-\varphi_{o 1}=\rho_{i}-\rho_{1}
\end{gathered}
$$

The only influence factors in (11) and (12) are the ratio error $\varepsilon_{i}$ and the phase error $\rho_{i}$ of CVTs respectively, the fluctuation of the primary voltage has been eliminated. Both the ratio error and phase error of CVTs are stable and not affected by power system during the normal operation, and it is almost impossible that multiple CVTs be malfunction at the same time. Therefore, if the internal insulation faults happen to the i-th CVT, its actual ratio error may deviate from the nominal values, then the two related ratios $V_{o(i-1)} / V_{o i}$ and $V_{o i} / V_{o(i+1)}$ in (11) would be disturbed, and these two ratios would varies in opposite directions respectively, the corresponding abnormal CVT can be recognized no matter how the power grid fluctuates. Similarly, the phase differences between the adjacent two CVTs $\varphi_{o(i-1)}-\varphi_{o i}$ and $\varphi_{o i}-\varphi_{o(i+1)}$ in (12) are determined by the actual phase error of each CVT, the groups of phase difference could embody the faulted CVT if $\rho_{i}$ is influenced by the fault.

\section{Proposed approach description}

As discussed in Section III A 2, off-line modeling and online monitoring are two main parts of the fault identification method. The specific procedure of the internal insulation faults identification is shown in Fig. 4 and discussed as follows.

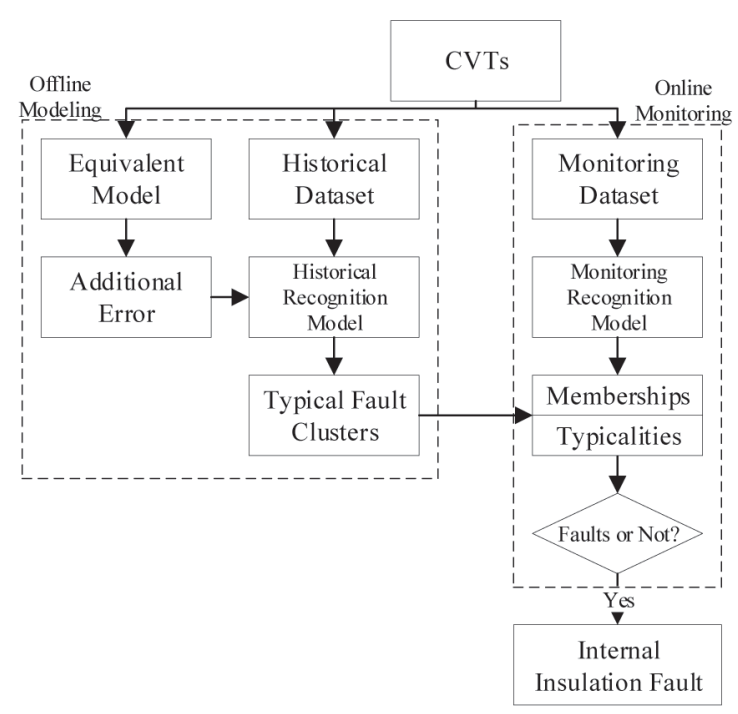

FIG. 4. Internal insulation fault identification procedure

First, the offline modeling:

1. Historical Dataset Composing: When the monitored CVTs are operating in the normal condition, the amplitude and phase of their output voltage are acquired to compose the historical dataset $X=\left[X_{1}, X_{2}, X_{3}, \ldots, X_{k}\right]$, $X_{k}$ contains amplitude and phase of the No.k CVT, all the CVTs in $X$ are connected to the same bus.

2. Additional Error Generation: Let $c$ be the quantity of the type of preset typical faults, the additional error is obtained through modifying the parameters of the simulation model in Fig. 2. 
3. Historical Model Generation: The dataset $X=$ $\left[X_{i-1}, X_{i}, X_{i+1}\right]$ is used to build the recognition models for the No.i CVT, then $c$ copies of $X$ are generated, each kind of additional error would be added to a duplicate dataset of $X_{i}$. The specific modeling method is introduced in Section III B 1, and the historical recognition model is formed with $V_{i-1} / V_{i}, V_{i} / V_{i+1}, \varphi_{i-1}-\varphi_{i}$, $\varphi_{i}-\varphi_{i+1}$ of each copy dataset.

4. Typical Fault Cluster Obtaining: The historical recognition model used to generate the typical fault clusters is:

$$
M_{h}=\left[\begin{array}{cccc}
V_{i-1}^{0} / V_{i}^{0} & V_{i}^{0} / V_{i+1}^{0} & \varphi_{i-1}^{0}-\varphi_{i}^{0} & \varphi_{i}^{0}-\varphi_{i+1}^{0} \\
V_{i-1}^{1} / V_{i}^{1} & V_{i}^{1} / V_{i+1}^{1} & \varphi_{i-1}^{1}-\varphi_{i}^{1} & \varphi_{i}^{1}-\varphi_{i+1}^{1} \\
\cdots & & & \\
V_{i-1}^{c} / V_{i}^{c} & V_{i}^{c} / V_{i+1}^{c} & \varphi_{i-1}^{c}-\varphi_{i}^{c} & \varphi_{i}^{c}-\varphi_{i+1}^{c}
\end{array}\right]
$$

Where the superscript 0 indicates that the model is in normal condition. The objective function (4) is built and the centers of $c+1$ typical clusters are obtained by PFCM. Besides, $c+1$ memberships and $c+1$ typicalities are also obtained, which indicate the clustering quality.

Then, the on-line monitoring procedures:

1. Monitoring Dataset Composing: After composing the historical dataset, the monitored CVTs turn into the monitoring status, the amplitude and phase of their output voltage are collected as the monitoring dataset, the structure of the dataset is completely same as the historical dataset.

2. Monitoring Model Generation: The monitoring dataset is used to build the monitoring model, and the structure of the model is:

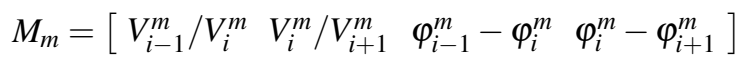

Where the superscript $m$ means the "monitoring".

3. Faults Indicators Calculation \& Faults Judgement: As discussed in Section III A 2, the insulation faults are indicated by memberships $u_{i k}$ and typicalities $t_{i k}$, calculating these two indicators of the monitoring models, which are relative to the $c+1$ historical clusters, then the insulation condition can be judged as Fig. 3 .

\section{CASE STUDY}

\section{A. Case Description}

In order to verify the effectiveness of the proposed insulation faults identification method in this paper, a monitoring system equipped with voltage acquisition device has been installed in a $220 \mathrm{kV}$ step-up substation. There are 6 voltage acquisition channels in the system, and each channel is responsible for a set of three-phase CVT, each set of monitored CVT
TABLE I. Typical specific parameters of CVTs

\begin{tabular}{cc}
\hline \hline Parameter & Value \\
\hline Rated Insulation voltage/kV & $395 / 950$ \\
HV Capacitor $/ \mu \mathrm{F}$ & 0.00367 \\
$n_{H}$ & 178 \\
$\tan \delta_{\mathrm{H}} / \%$ & 0.079 \\
$\mathrm{MV} \mathrm{Capacitor} / \mu \mathrm{F}$ & 0.033 \\
$n_{M}$ & 20 \\
$\tan \delta_{\mathrm{M}} / \%$ & 0.075 \\
$\mathrm{~L}_{\mathrm{k}} / \mathrm{H}$ & 274 \\
$\mathrm{R}_{\mathrm{m}} / \mathrm{k} \Omega$ & 15488 \\
$\mathrm{X}_{\mathrm{m}} / \mathrm{k} \Omega$ & 42530 \\
$\mathrm{R}_{1}, \mathrm{R}_{2} / \mathrm{k} \Omega$ & 4 \\
$\mathrm{X}_{\mathrm{T} 1}, \mathrm{X}_{\mathrm{T} 2} / \mathrm{k} \Omega$ & 676 \\
\hline \hline
\end{tabular}

are connected to a three-phase outgoing lines, so all the monitored CVTs have the same primary voltage as the busbar, the simplified connecting diagram of the substation is shown in Fig. 5 (C1 $\cdots$ C6 are six groups of three-phase CVT, T1 $\cdots$ T4 are four main transformers, ST1 and ST2 are two spare transformers), all the breakers are closed during the monitoring. The accuracy class of the acquisition device is 0.05 , which means its maximum permitted ratio error is $\pm 0.05 \%$, and the maximum permitted phase error is $\pm 2^{\prime}$. The accuracy class of the monitored CVTs is 0.2 , and the nominal primary is 220 $\mathrm{kV}$, the rated output phase voltage is $100 / \sqrt{3} \mathrm{~V}$, the typical specific parameters of these CVTs is shown in Table. I.

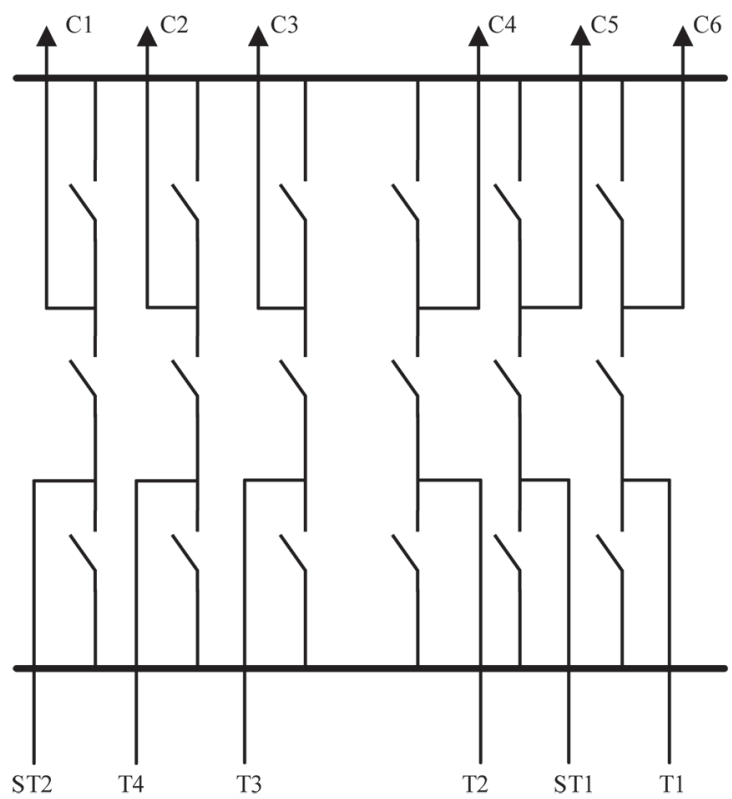

FIG. 5. The simplified connection diagram

The voltage acquisition lasted for more than one month, and the system store a data point every 15-minute, which contains the amplitude and phase of the output voltage of all the monitored CVTs. The phase A of the No. 4 CVT 
TABLE II. Specific typical faults in historical dataset

\begin{tabular}{cc}
\hline \hline Samples & Condition \\
\hline $1 \sim 1000$ & Normal \\
$1001 \sim 2000$ & 5 capcitors out \\
$2001 \sim 3000$ & 10 capcitors out \\
$3001 \sim 4000$ & $0.2 \%$ dielectric loss \\
$4001 \sim 5000$ & $0.5 \%$ dielectric loss \\
\hline \hline
\end{tabular}

is taken as example, 3000 samples are selected as the testing dataset, and the first 1000 samples are selected as the historical dataset, the recognition models $V_{3 A} / V_{4 A}, V_{4 A} / V_{5 A}$, $\varphi_{3 A}-\varphi_{4 A}$ and $\varphi_{4 A}-\varphi_{5 A}$ based on the monitoring dataset is shown in Fig. 6. Obviously, all the fluctuations in the power system have been eliminated.

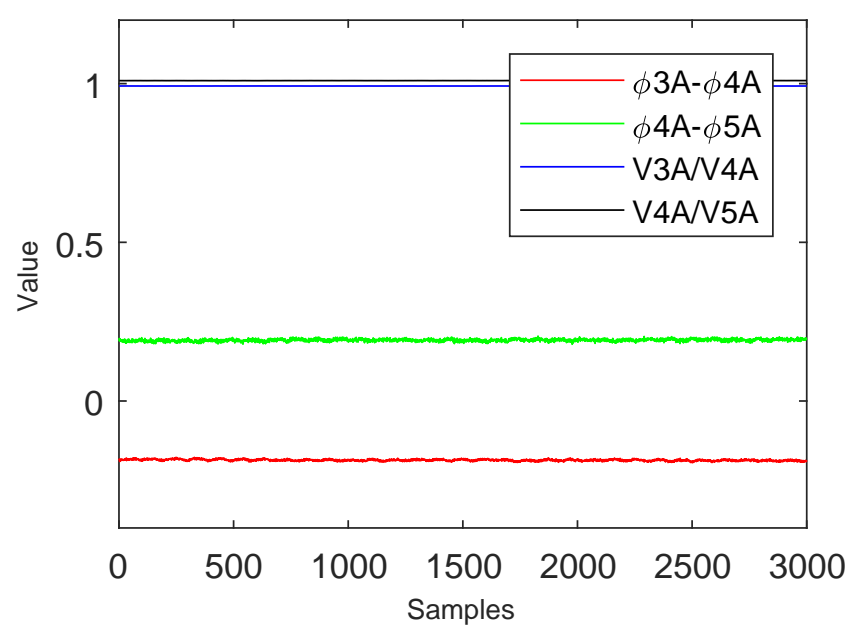

FIG. 6. Recognition models of the phase A of No. 4 CVT

The additional errors of several typical faults are added to copies of the monitoring dataset to generate the typical clusters. Since the number of $\mathrm{HV}$ capacitor is much more than MV capacitor, same faults occur to MV capacitor have greater influence on the measurement error than HV capacitor, the clustering algorithm could identify the faults of MV capacitor more easily. Therefore, in this Section, the typical faults considered to verify the effectiveness of the proposed method are only HV capacitor breakdown and HV capacitor excessive dielectric loss, each kind of typical fault consists of two different fault degrees, additional error of these faults is added to the corresponding 4 copies of the historical dataset, thus there are 5000 samples in the historical dataset, the specific faults in the historical dataset is shown in Table II. The typical fault clusters are generated by PFCM, the memberships and typicalities of each cluster are shown in Fig. 7.

In Fig. 7, each membership and typicality curve corresponds to a typical fault cluster, the maximum membership of each segment of samples is 1 , and the typicalities reach the optimal value (In PFCM, $a=2, b=3, m=\eta=2$, the optimal value of typicality is 0.25 ), compared with the Table II, both the membership and typicality indicate that the historical dataset has been clustered clearly, then center of each cluster is also obtained.

\section{B. Case1: Fault degree less than the maximum typical faults}

In this section, the $1001 \sim 1500$ samples are selected as the testing dataset, some typical faults are added to parts of the samples. Since the degree of typical faults in the historical dataset is not continuous, the fault setting of the testing dataset in this section consists of typical faults and faults between two adjacent typical faults in Table. II.

\section{Capacitors breakdown}

Firstly, assuming that there are $5 \mathrm{HV}$ capacitors be broken down during the operation at the 200th testing data point, which means the first 200 testing data points should belong to the normal operation condition and the following 300 testing data points should be classified to the corresponding typical fault cluster.

The corresponding parameters of the equivalent model are changed to calculate the additional error, these errors are added to the faulted part of the testing dataset. Calculating the memberships and typicalities of the testing dataset, each membership and typicality corresponds to a typical fault cluster. The memberships and typicalities of the testing dataset are shown in Fig. 8.

In Fig. 8, each curve corresponds to one of the typical conditions in Table. II successively. When the monitored CVT is still operating in the normal condition, the first membership curve (corresponding to the normal condition) is 1 while other membership curves are close to zero. Besides, the first typicality curve is 0.25 while other typicality curves are closed to zero. Both the membership and typicality of the first 200 testing samples indicate the right identification result. The following 300 monitored data points contain the 5 capacitors breakdown fault information, then the first membership curve turns to zero while the second membership curve (corresponding to the 5 capacitors breakdown fault) switches from 0 to 1 at the same time, which means the fault identification method recognised the fault in testing dataset correctly and immediately, there are 5 broken HV capacitors in the monitored CVT.

Then, assuming that there are $3 \mathrm{HV}$ capacitors be broken down at the 200th testing data point, the degree of the fault is between the normal condition and the first typical typical fault in the historical dataset. The memberships and typicalities of this testing dataset are shown in Fig. 9.

In Fig. 9, the first membership curve turns to almost 0 at the 200th data point, which is similiar to Fig. 8. Meanwhile, the second membership curve turns to about 0.8 . Be subject to the constraint $\sum_{i=1}^{c} u_{i k}=1$, as the second membership does not reach 1 , the third and the fourth membership curves are a little larger than 0 from the 200th data point. Since the second membership is much larger than others, the membership curves indicate that the second typical fault is most likely happened to the monitored CVT, but the degree of the fault 


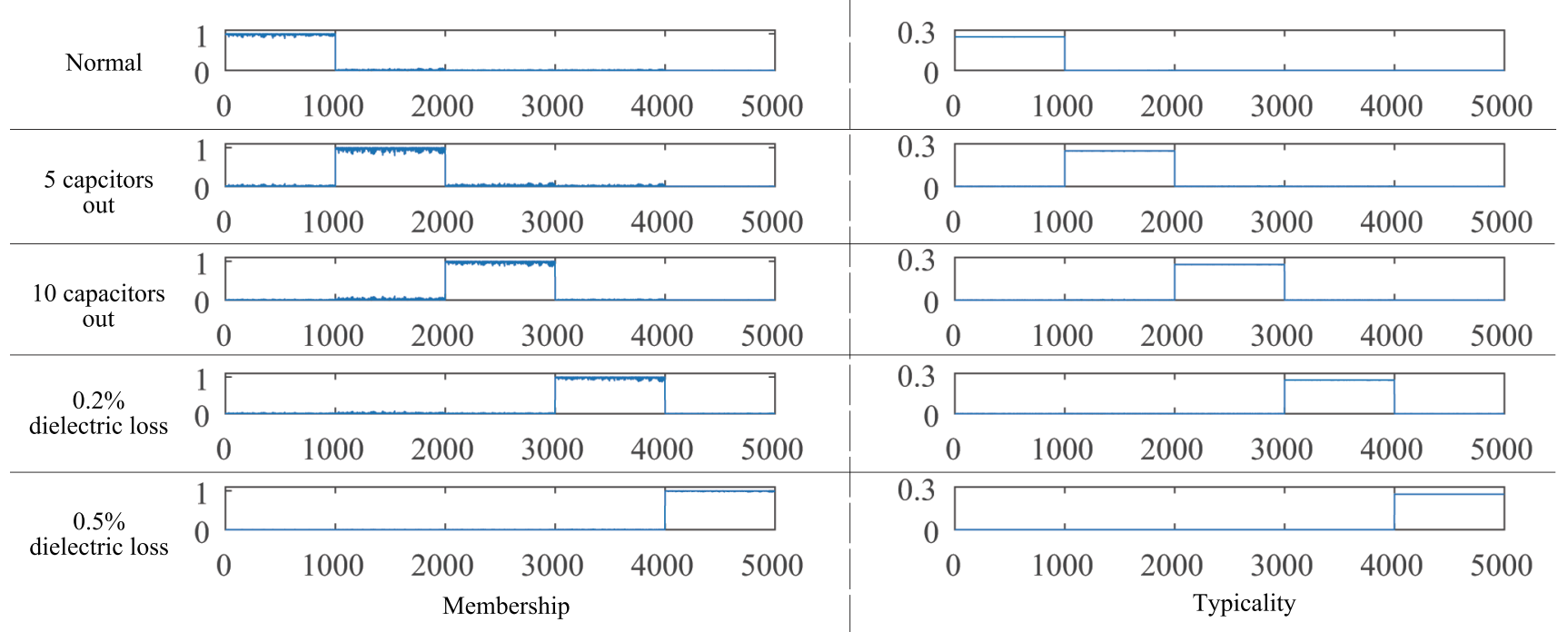

FIG. 7. Memberships and typicalities of the historical dataset

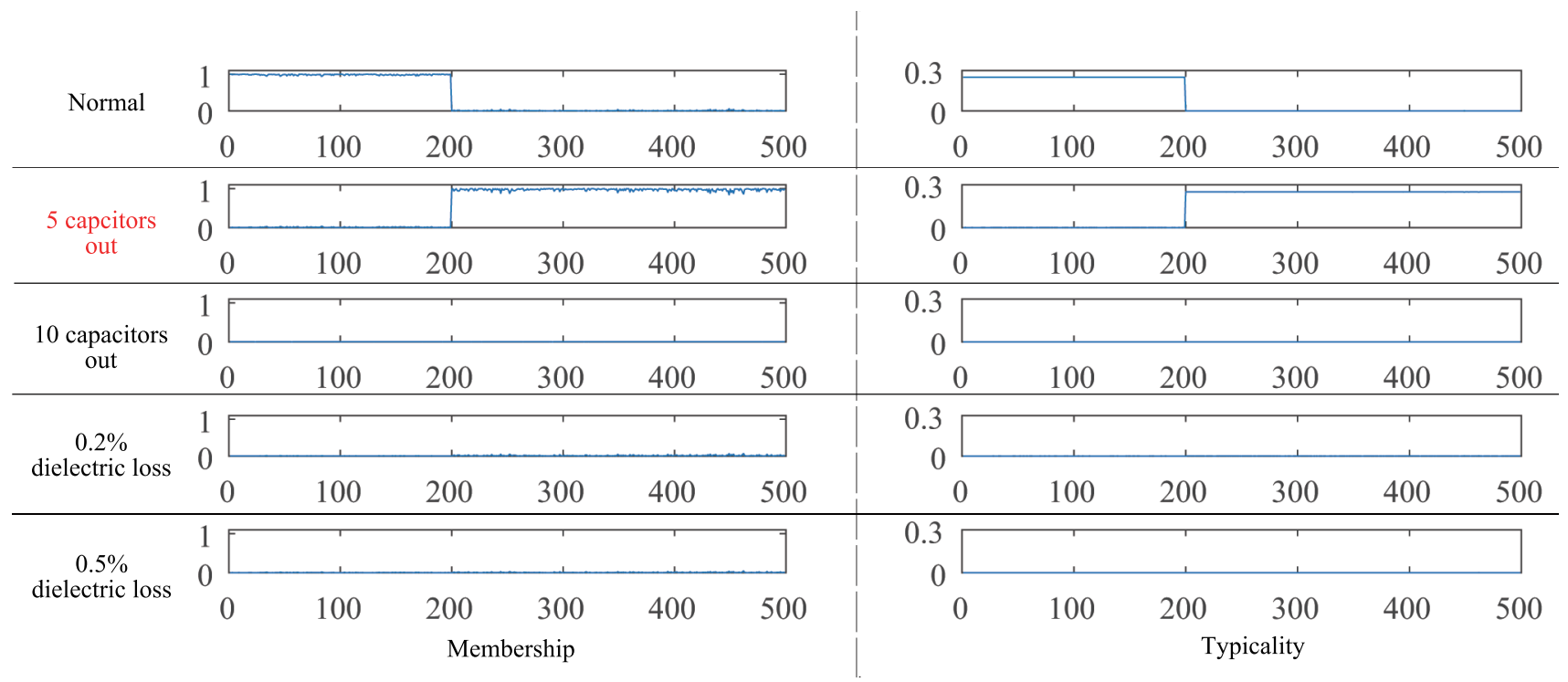

FIG. 8. Memberships and typicalities of 5 capacitors breakdown in testing dataset

should be less than the second typical fault. As to the typicality curves in Fig. 9, the second typicality curve almost reaches the optimal value from the 200th data point, and there is no variation happens in the $3 r d \sim 5 t h$ curve at this moment. So both the memberships and the typicalities indicate the same conclusion, the monitored CVT is operating in fault condition, and the typical fault corresponding to the second curve is most likely to occur, the degree of the fault in the testing dataset should be closed to second typical fault, which is consistent with reality.

\section{Excessive dielectric loss}

Similar to the section IV B 1, the excessive dielectric loss faults in the testing dataset is divided into typical fault in historical dataset and fault between two adjacent typical faults. The additional errors generated by the equivalent model are added to the testing dataset.

Assuming that the excessive dielectric loss tangent of HV capacitors is $0.2 \%$ and $0.3 \%$ respectively, the memberships and typicalities of the testing dataset are shown in Figs. 10 and 11. Both these two faults could be identified by the proposed method.

In the Fig. 10, The first membership curve turns to zero at the 200th data point, while the fourth membership curve turns 


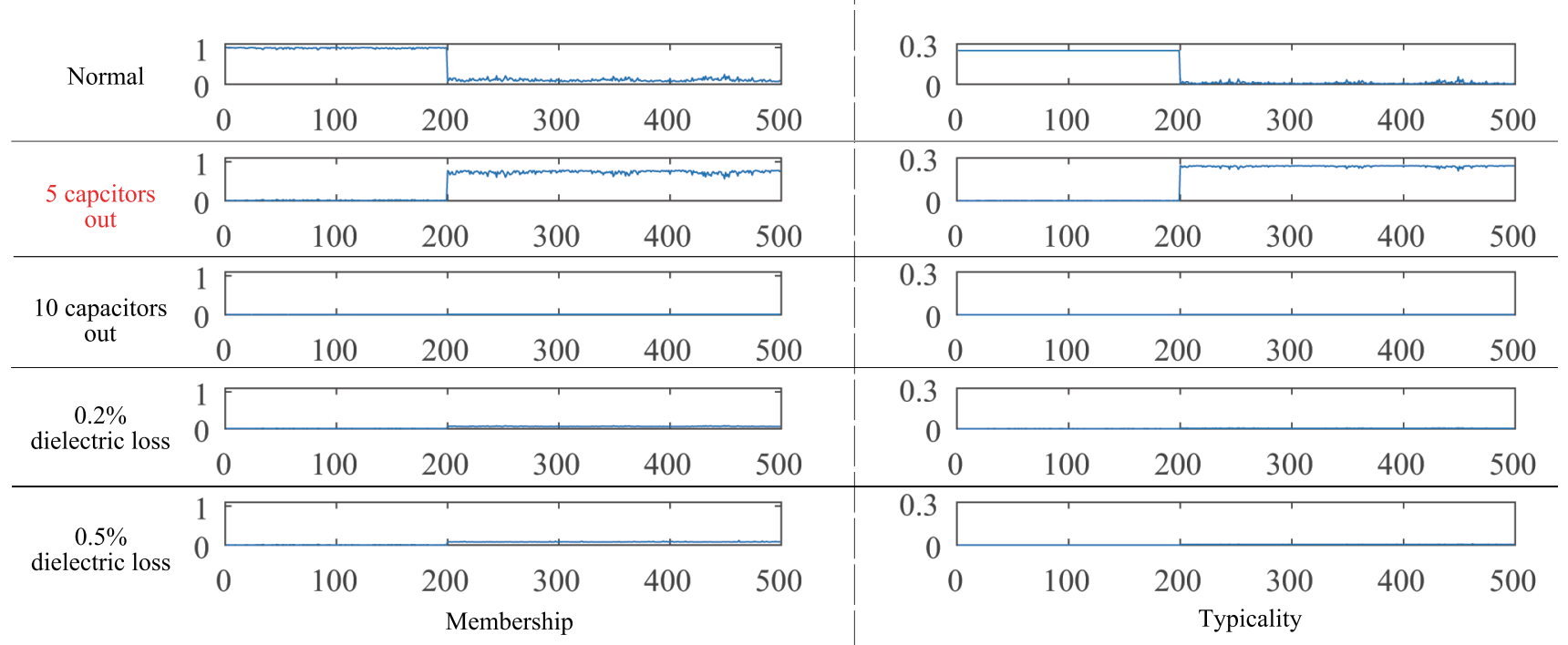

FIG. 9. Memberships and typicalities of 3 capacitors breakdown in testing dataset

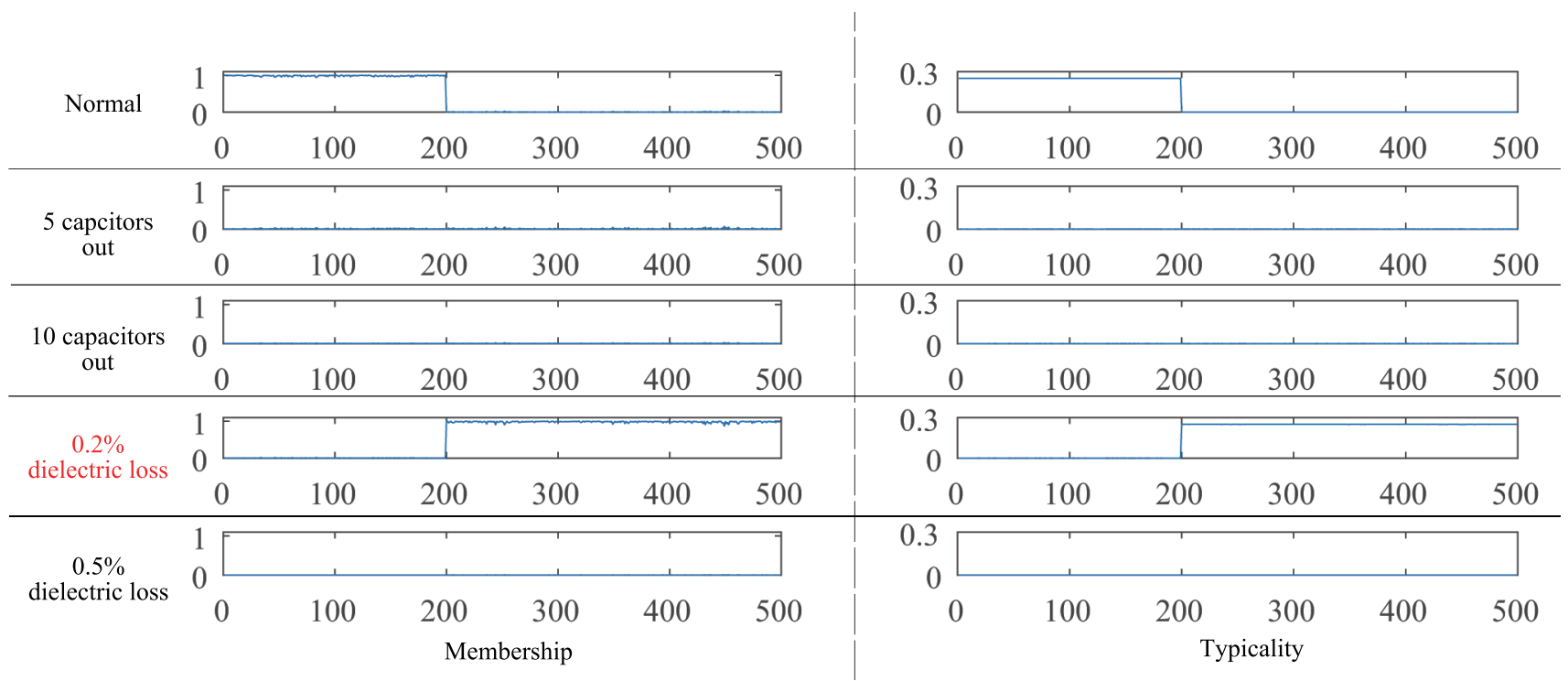

FIG. 10. Memberships and typicalities of $0.2 \%$ dielectric loss tangent in testing dataset

to 1 , all the other membership curves remain zero. Besides, the first typicality curve turns to zero at the 200th data point, and the fourth typicality curve turns to the optimal value at the same time, other typicality curves also remain zero. So it can be concluded that the dielectric loss of the monitored CVT reaches $0.2 \%$. In Fig, 11, the membership curve corresponding to $0.2 \%$ dielectric loss tangent is lower than 1 from the 200th data point, and all the other membership curves are slightly larger than zero. Besides, the fourth typicality curve also indicates that the dielectric loss of the monitored CVT is most likely $0.2 \%$, while all the other typicality curves remain zero, but the curve corresponding to the $0.5 \%$ dielectric loss tangent is slightly larger than $1 s t \sim 3 r d$ typicality curves. Therefore, it can conclude from this figure that excessive di- electric loss has happened to the monitored CVT, and the fault degree is slightly larger than $0.2 \%$. So, according to Figs. $10 \sim 11$, both the memberships and the typicalities could indicate the excessive dielectric loss fault if the degree of fault is lower than the maximum typical faults.

\section{Case2: Fault degree beyond the maximum typical faults}

In this case, the $1001 \sim 1500$ samples are still selected as the testing dataset, the insulation faults beyond the maximum typical faults are considered to test the adaptability of the proposed method. Therefore, the additional errors of $1 \%$ and $2 \%$ dielectric loss tangent of $\mathrm{HV}$ capacitors are calculated and 


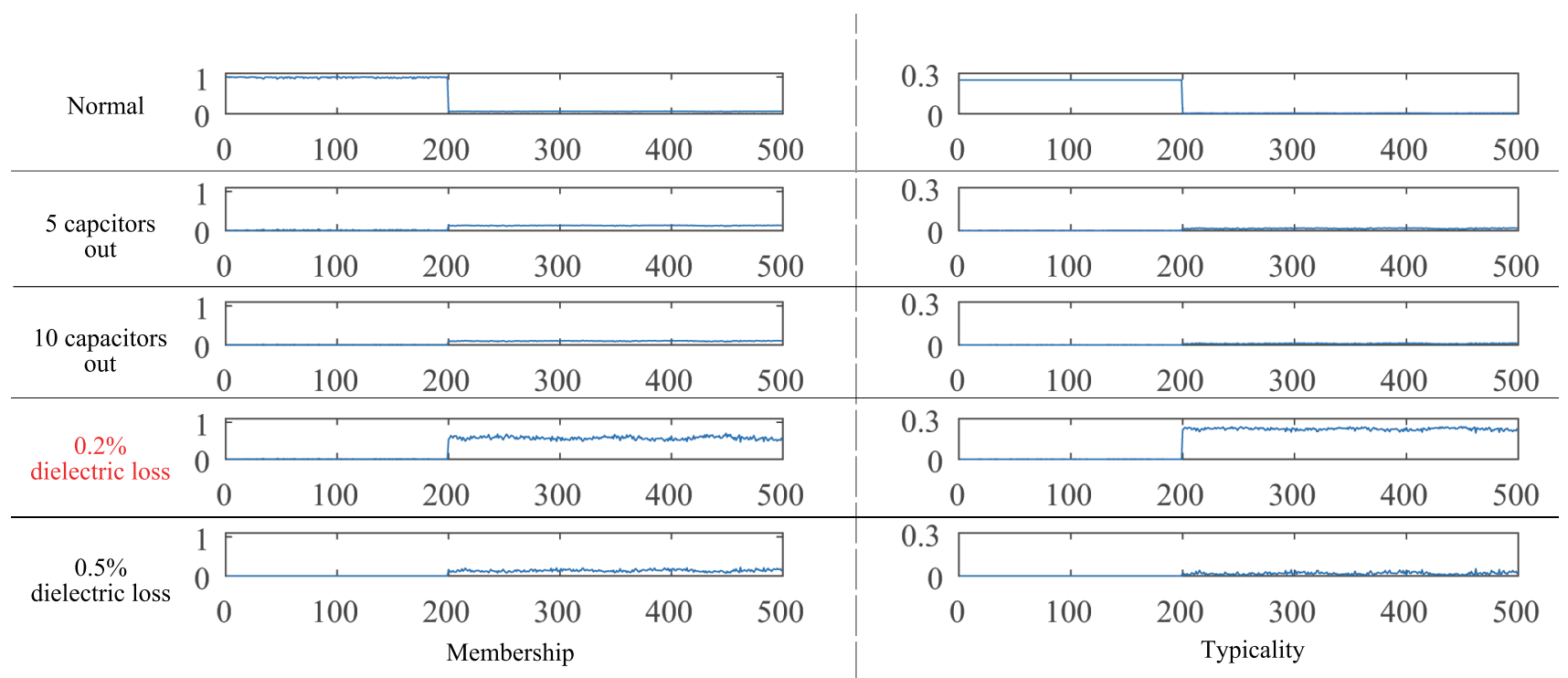

FIG. 11. Memberships and typicalities of $0.3 \%$ dielectric loss tangent in testing dataset

added to the faulted part of the testing dataset respectively, the degree of both these two faults are greater than the maximum dielectric loss fault in Table II. The memberships and typicalities of the testing dataset in these two fault conditions are shown in Figs. 12 and 13 respectively.

According to the constraint $\sum_{i=1}^{c} u_{i k}=1$, each $u_{i k}$ would be $1 / 5$ instead of zero if the testing dataset does not belong to any cluster of the historical dataset. In Figs. 12 and 13, all the membership curves switch to a nonzero value at the 200th data point, the last membership is sightly larger than others in Fig. 12, and it becomes more closer to $1 / 5$ in Fig. 13 since the degree of fault in Fig. 13 is further to the maximum preset typical dielectric loss fault than that in Fig. 12. In this case, memberships are meaningless for fault identification. As to the typicalities, the first typicality curve in Fig. 12 turns to zero at the 200th data point while the 2 nd $\sim 4$ th typicality curves stay closed to almost zero, and the last typicality curve switches from zero to a larger value, which is still less than the optimal value. Similarily, the first typicality curve also turns to zero at the 200th data point in Fig. 13, the 2nd $\sim 4$ th typicality curves remain zero either, the value of the last typicality curve also switches to a larger value, but the value is less than that in Fig. 12. Therefore, it can be concluded that excessive dielectric loss faults have happened to the monitored CVT, and the degree of the faults are larger than the maximum faults in the historical dataset. What's more, since the typicality curve corresponding to the maximum typical fault in Fig. 12 is larger than that in Fig. 13, the fault in Fig. 12 is closer to the maximum typical fault than that in Fig. 13, which is matched with the facts.

Combining the results of Case 1 and Case 2, no matter the degree of fault is beyond the maximum preset typical faults or not, the proposed method could identify that the monitored CVT is not operating properly. If the fault degree is less than the maximum typical faults, the approximative fault degree could be obtained by the proposed method either, if the fault degree is beyond the maximum typical faults, the proposed method is able to give a fault warning in time.

\section{CONCLUSIONS}

With the increment of the voltage level of modern power system, the inaccuracy measurement, the power failure and even the explosion caused by the insulation faults of CVTs would lead to serious consequences. Based on the possibilistic fuzzy c-means clustering method, this paper proposed an approach to monitor the internal insulation condition and identify the insulation faults of CVTs.

The proposed method doesn't need any extra equipment to collect other parameters but the amplitude and phase of the output voltage, it combines the output voltages of CVTs with their distribution topology, then stable recognition models for fault identification are built. These models are not affected by the fluctuations of the power system, and the only influence factor of the model is the measurement error of CVTs, which is related to the internal insulation condition. The PFCM is used to cluster the recognition models, and the memberships and typicalities of the monitoring dataset determine which cluster the monitored dataset belongs to, then the insulation condition of the monitored CVT is obtained.

Some cases in Section IV illustrate that the proposed method could identify the internal insulation faults if the fault degree is less than the maximum preset typical faults. If the faults are beyond the preset typical faults, the proposed method could also recognize that the monitored CVT is not operating in the normal condition. 


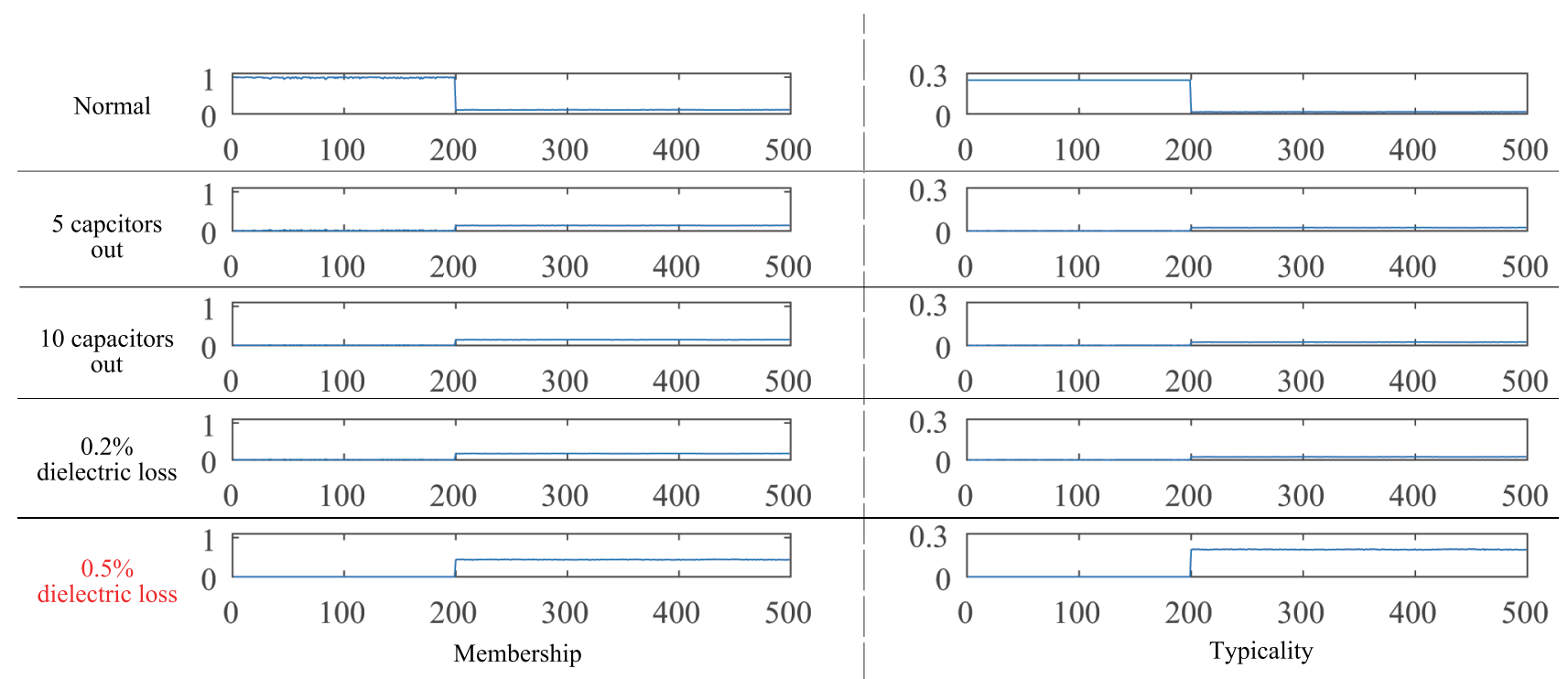

FIG. 12. Membership and typicality of $1 \%$ dielectric loss tangent in testing dataset

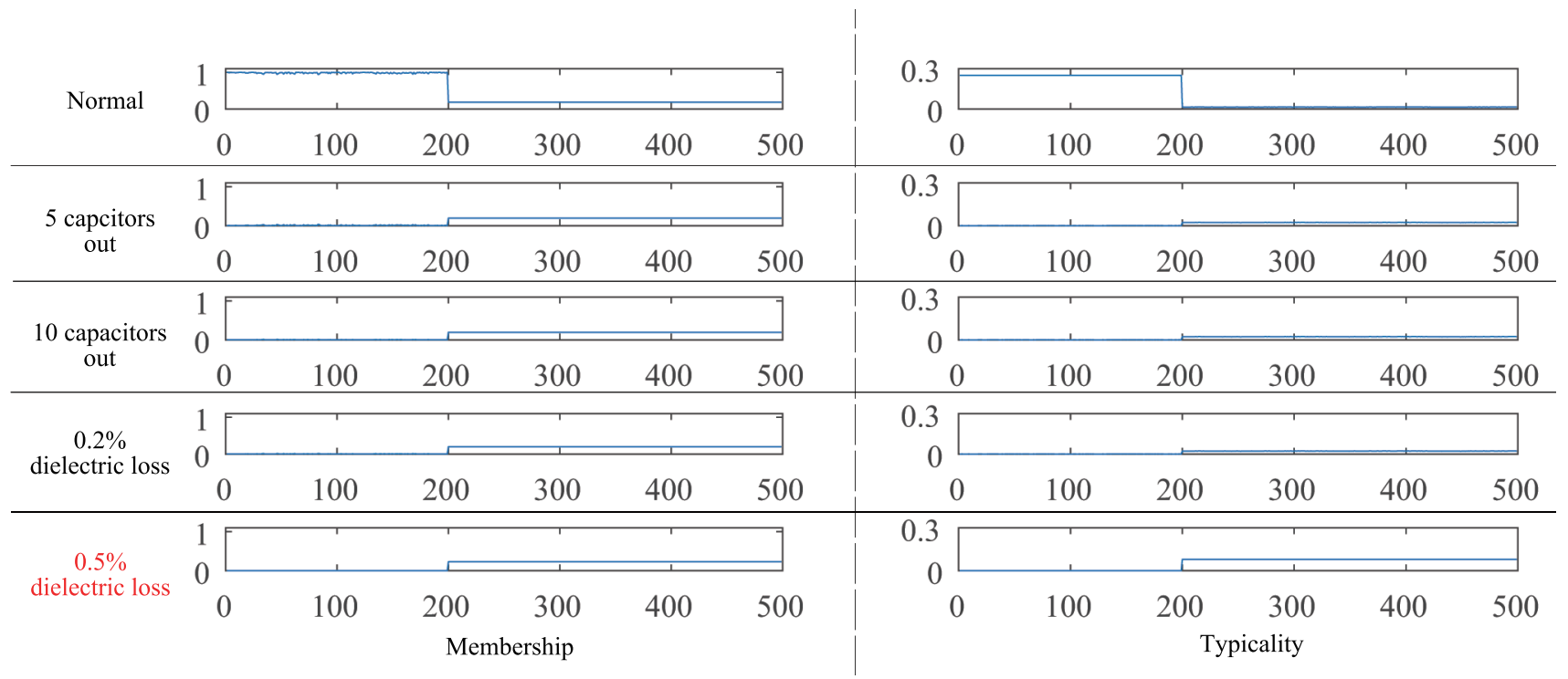

FIG. 13. Membership and typicality of $2 \%$ dielectric loss tangent in testing dataset

\section{ACKNOWLEDGMENTS}

This work is supported by the National Natural Science Foundation of China (Grant NO.U1866201).

\section{REFERENCES}

${ }^{1}$ J. Le, Y. Liu, Q. Li, X. YU, and K. LIU, "Harmonic measurement error test technology of capacitor voltage transformer," Autom. Electr. Power Syst. 40, 108-113 (2016).

${ }^{2}$ D. Lin, C. Bin, and C. Weigen, "Research on the measurement error of capacitor voltage transformer under various insulation characteristics," in 2016 IEEE International Conference on High Voltage Engineering and Application (ICHVE) (IEEE, 2016) pp. 1-4.
${ }^{3}$ C. Zhang, H.-b. Li, J. Yang, M.-z. Chen, and Y. Jiao, "Detecting measurement error drifts of the capacitor voltage transformer on-line and its field application," Measurement Science and Technology (2019).

${ }^{4}$ Z.-H. Li, Y. Wang, Z.-T. Wu, and Z.-X. Li, "Research on electronic voltage transformer for big data background," Symmetry 10, 234 (2018).

${ }^{5} \mathrm{Z}$. Meng, H. Li, C. Zhang, M. Chen, and Q. Chen, "Research on the reliability of capacitor voltage transformers calibration results," Measurement 146, 770-779 (2019).

${ }^{6}$ A. Bakar, N. Rahim, and M. Zambri, "Analysis of lightning-caused ferroresonance in capacitor voltage transformer (cvt)," International Journal of Electrical Power \& Energy Systems 33, 1536-1541 (2011).

${ }^{7}$ L. W, D. L, and Y. Z., "On-site dielectric loss measurement method and error analysis of capacitor voltage transformer," Power Capacitor \& Reactive Power Compensation 3, 72-76 (2015).

${ }^{8} \mathrm{~L}$. Shun-Yao, "A test method for $220 \mathrm{kv}$ capacitive voltage transformer," High Voltage Apparatus 3 (2008). 
${ }^{9}$ J. H, X. C, and T. L. Fuzhou Z, "Design and application of on-line monitoring system for capacitor voltage transformer," Electrical measurement \& Instrumentation 53, 33-37 (2016).

${ }^{10}$ J. Li, Z. Teng, Q. Tang, J. Song, and X. Li, "Dielectric loss factor measurement in power systems based on sampling sequence reconstruction approach," IEEE Transactions on Power Electronics 32, 4766-4775 (2016).

${ }^{11}$ N. Daryani and H. Seyedi, "Evidence theory-based identification of aging for capacitive voltage transformers," IET Generation, Transmission \& Distribution 10, 3646-3653 (2016).

${ }^{12}$ B. Chen, L. Du, K. Liu, X. Chen, F. Zhang, and F. Yang, "Measurement er ror estimation for capacitive voltage transformer by insulation parameters," Energies 10 (2017).

${ }^{13}$ Y.-t. YAN, X.-f. NI, J.-w. JIANG, J.-1. ZHONG, and W.-j. GAN, "Theoretical analysis and experiment about effect of contamination resistance on accuracy of cvt," High Voltage Apparatus 2 (2009).

${ }^{14}$ M.-c. HE, J.-p. ZHANG, and J.-t. ZHAO, "Influence of the temperature changes on cvt's accuracy," Power Capacitor 1 (2006).

${ }^{15}$ L. D, B. C, X. C, F. Z, Y. L, and L. R., "Measurement errors of capacitor voltage transformer with different internal insulation parameters," Power System Technology. 40, 359-367 (2016).
${ }^{16} \mathrm{~J}$. HE, R. YU, and Y.-j. YANG, "The common faults and its monitoring of cvt [j]," Power Capacitor \& Reactive Power Compensation 5 (2009).

${ }^{17}$ H.-b. LI, Y.-b. LIU, G.-x. YE, and X.-q. WANG, "Numerical simulation on ferromagnetic resonances of capacitive voltage transformer," High Voltage Apparatus 2 (2004)

${ }^{18}$ IEC., IEC 61869-5: Instrument transformers-part 5: Additional requirements for capacitor voltage transformers (2011).

${ }^{19}$ B. J. C., Fuzzy mathematics in pattern classification, Ph.D. thesis, Cornel University (1973).

${ }^{20}$ J. C. Bezdek, R. Ehrlich, and W. Full, "Fcm: The fuzzy c-means clustering algorithm," Computers \& Geosciences 10, 191-203 (1984).

${ }^{21}$ R. Krishnapuram and J. M. Keller, “A possibilistic approach to clustering," IEEE transactions on fuzzy systems 1, 98-110 (1993).

${ }^{22}$ N. R. Pal, K. Pal, J. M. Keller, and J. C. Bezdek, "A possibilistic fuzzy cmeans clustering algorithm," IEEE transactions on fuzzy systems 13, 517530 (2005).

${ }^{23}$ Z. Zhang, H. Li, D. Tang, C. Hu, and Y. Jiao, "Monitoring the metering performance of an electronic voltage transformer on-line based on cyberphysics correlation analysis," Measurement Science and Technology 28, 105015 (2017)

${ }^{24}$ Y.-J. Kim, "Development and analysis of a sensitivity matrix of a threephase voltage unbalance factor," IEEE Transactions on Power Systems 33, 3192-3195 (2018). 

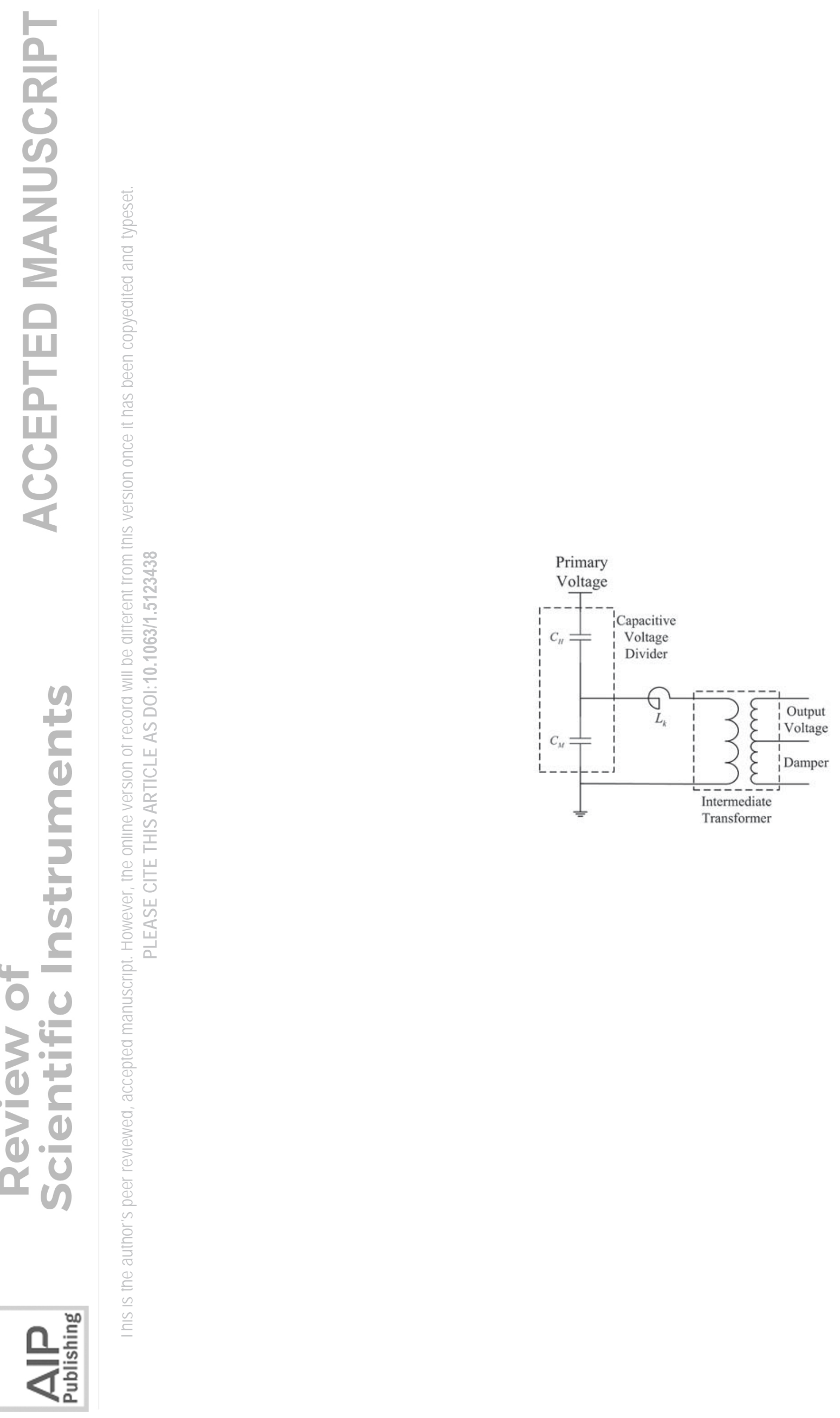

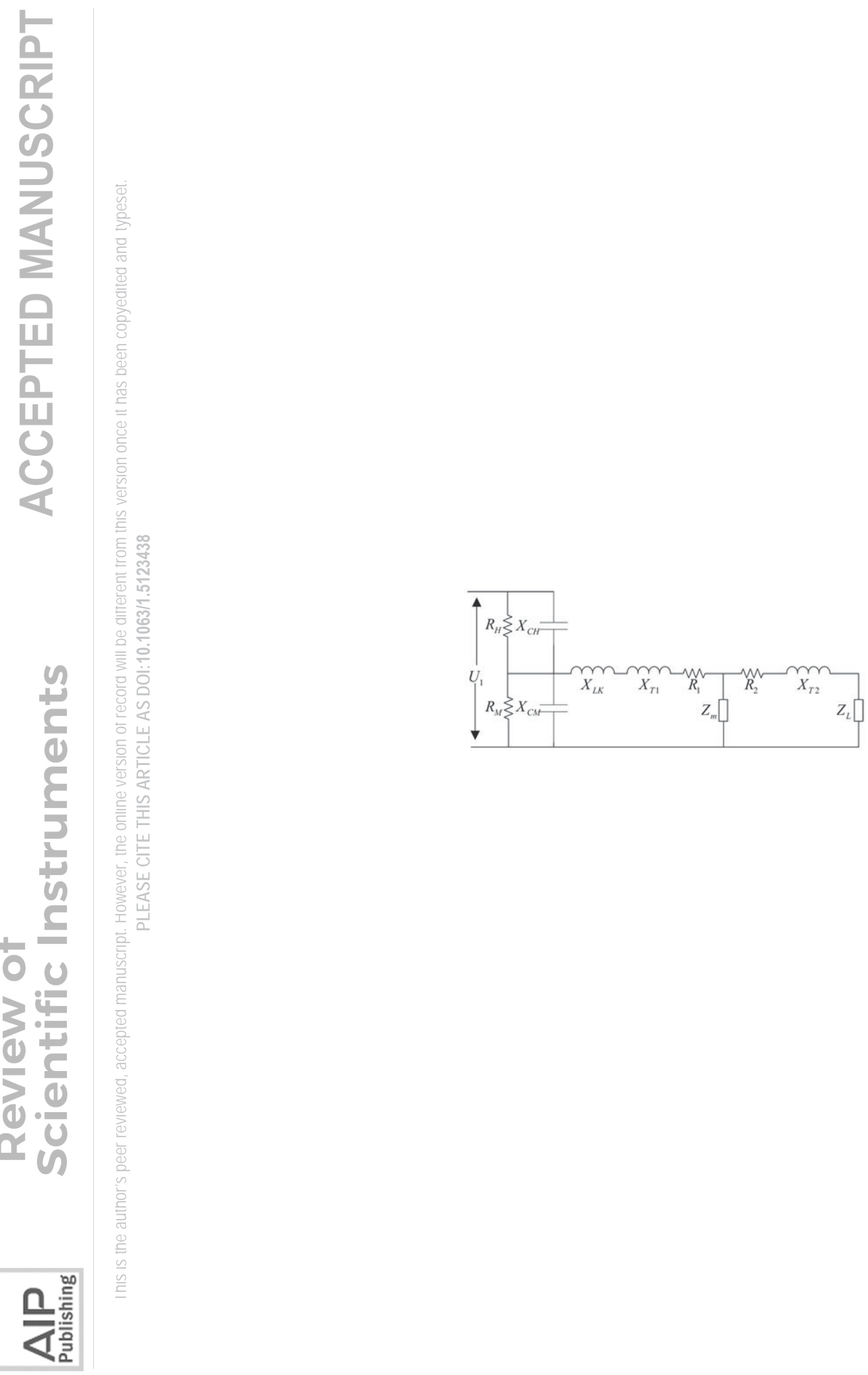


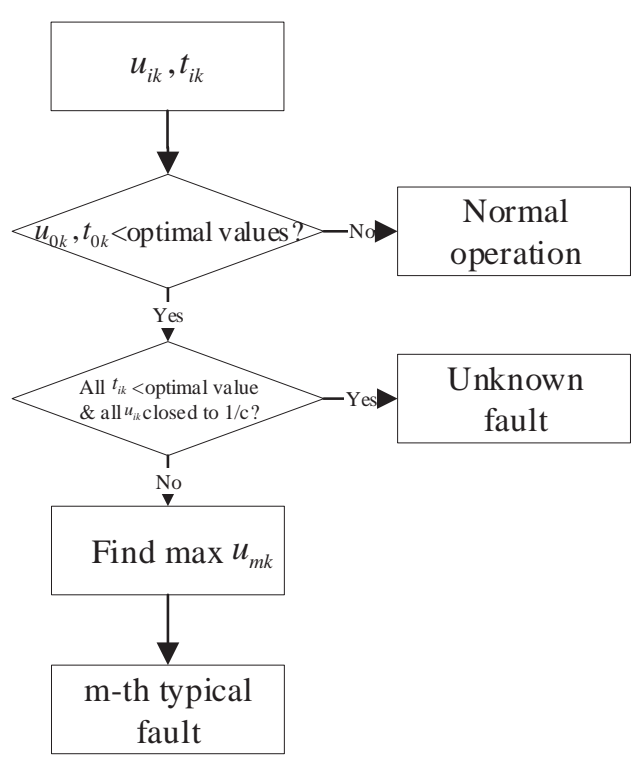



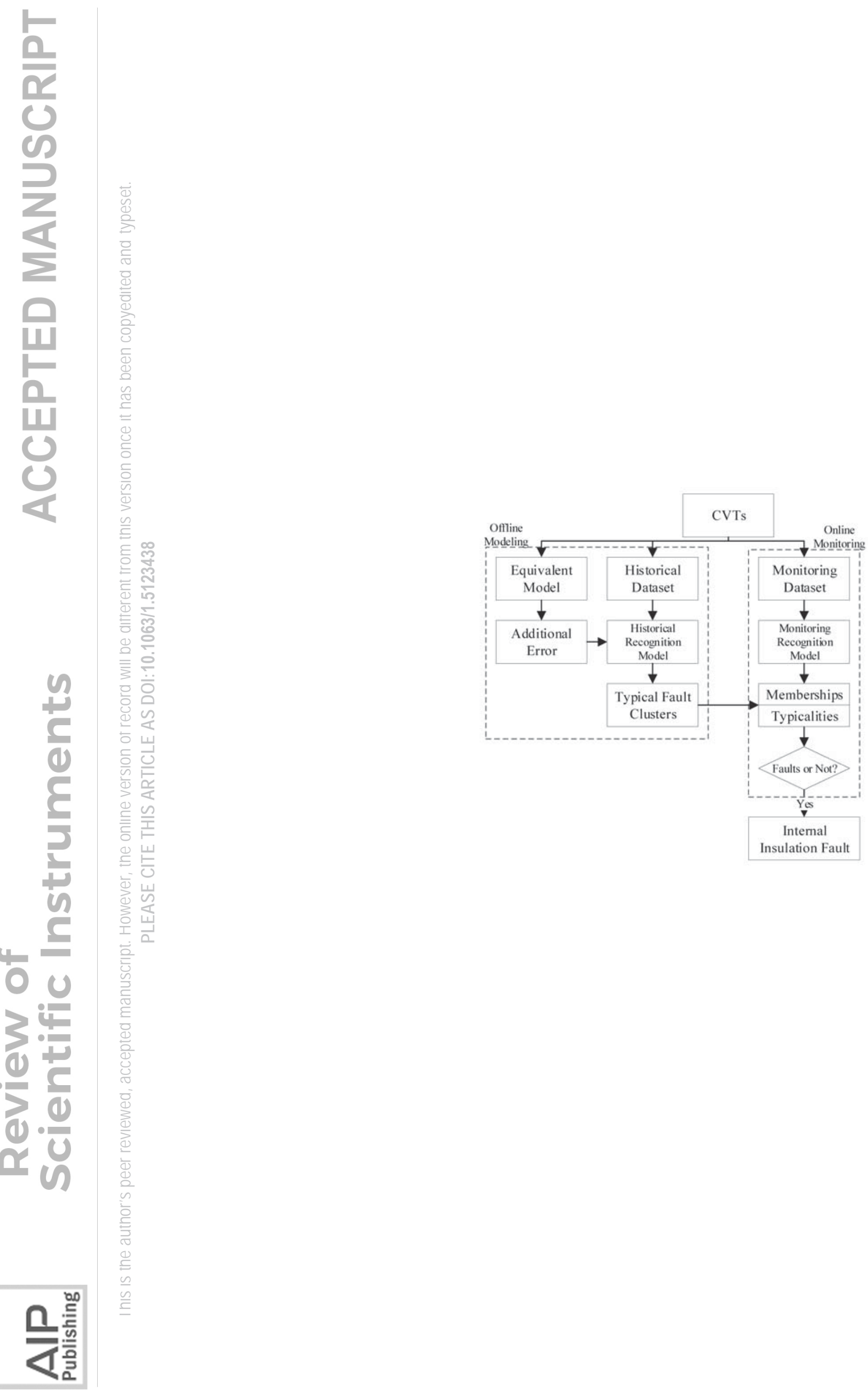

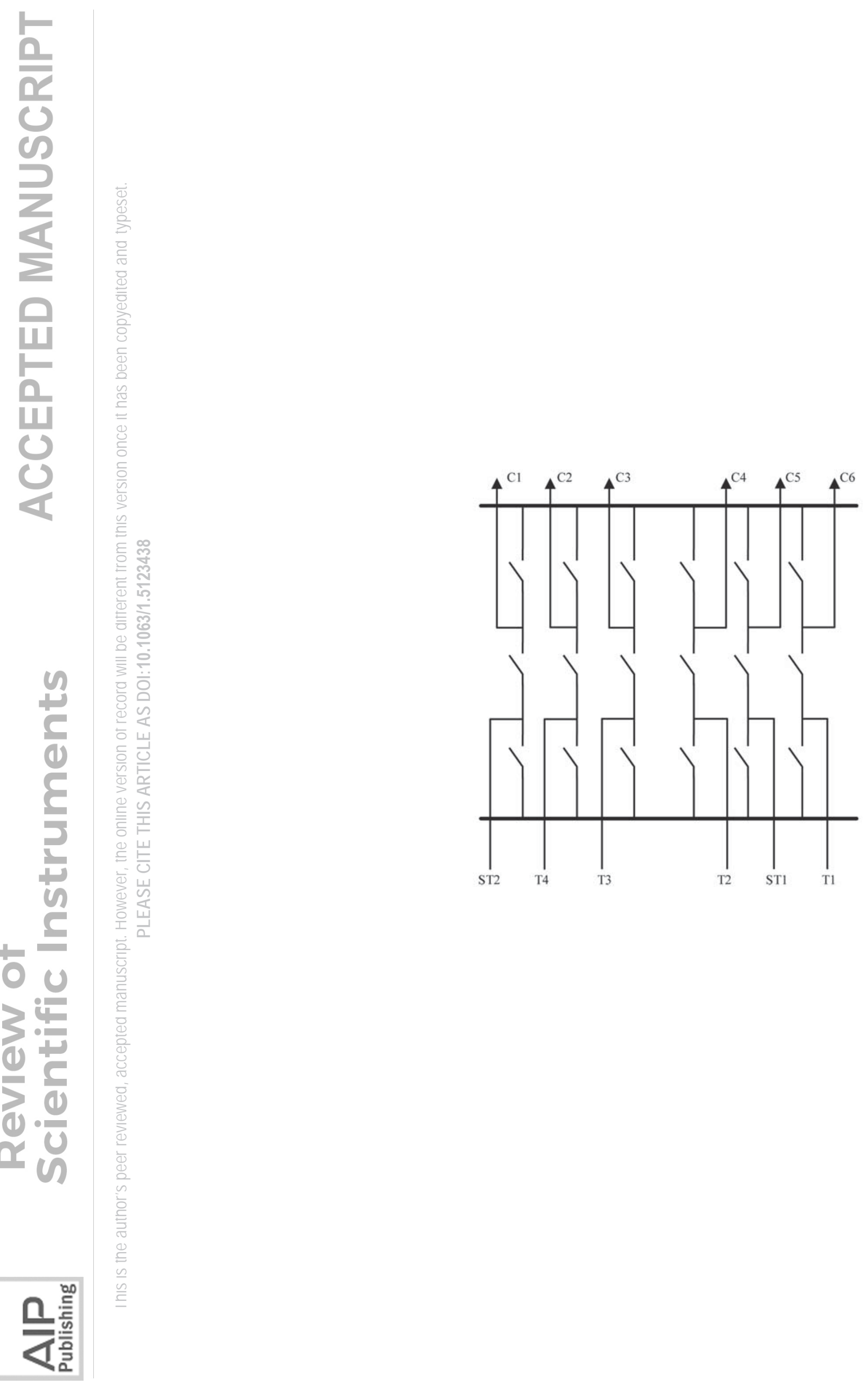


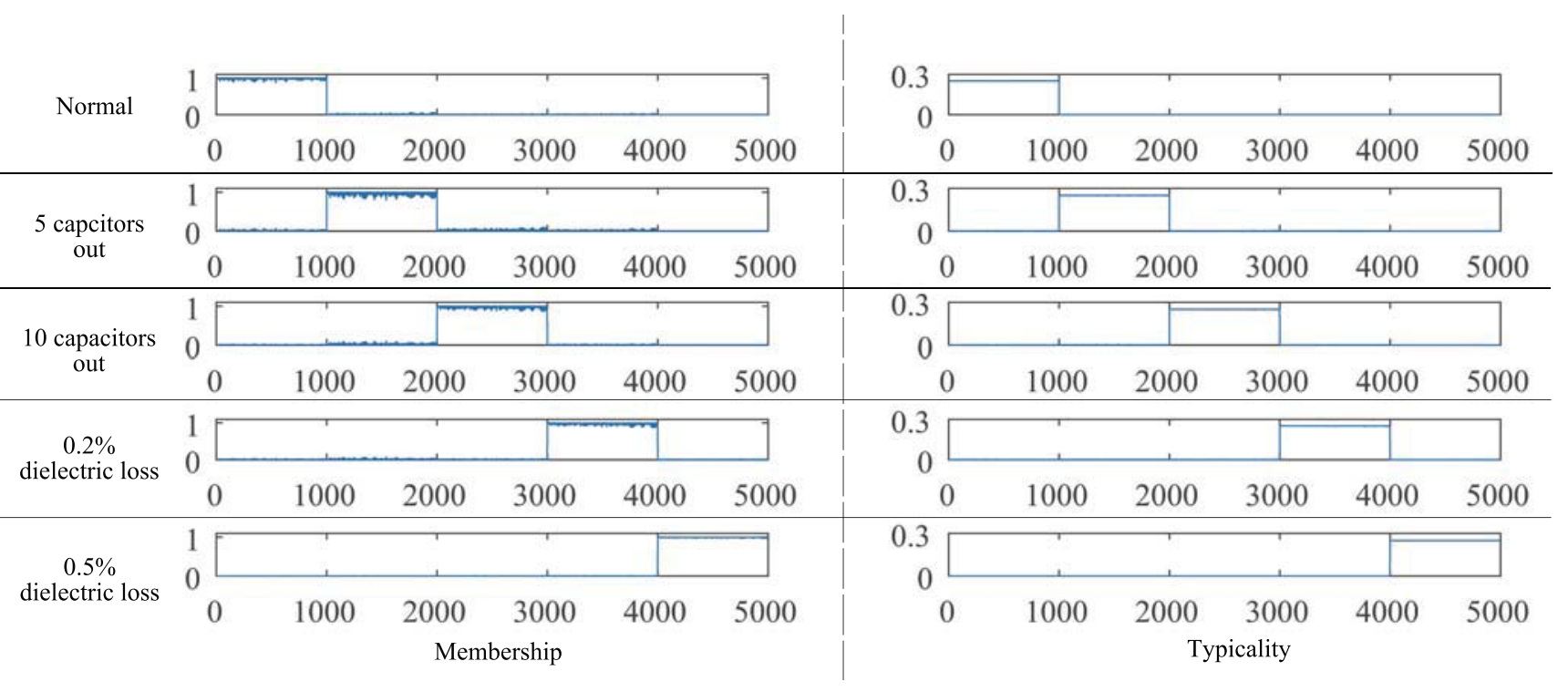




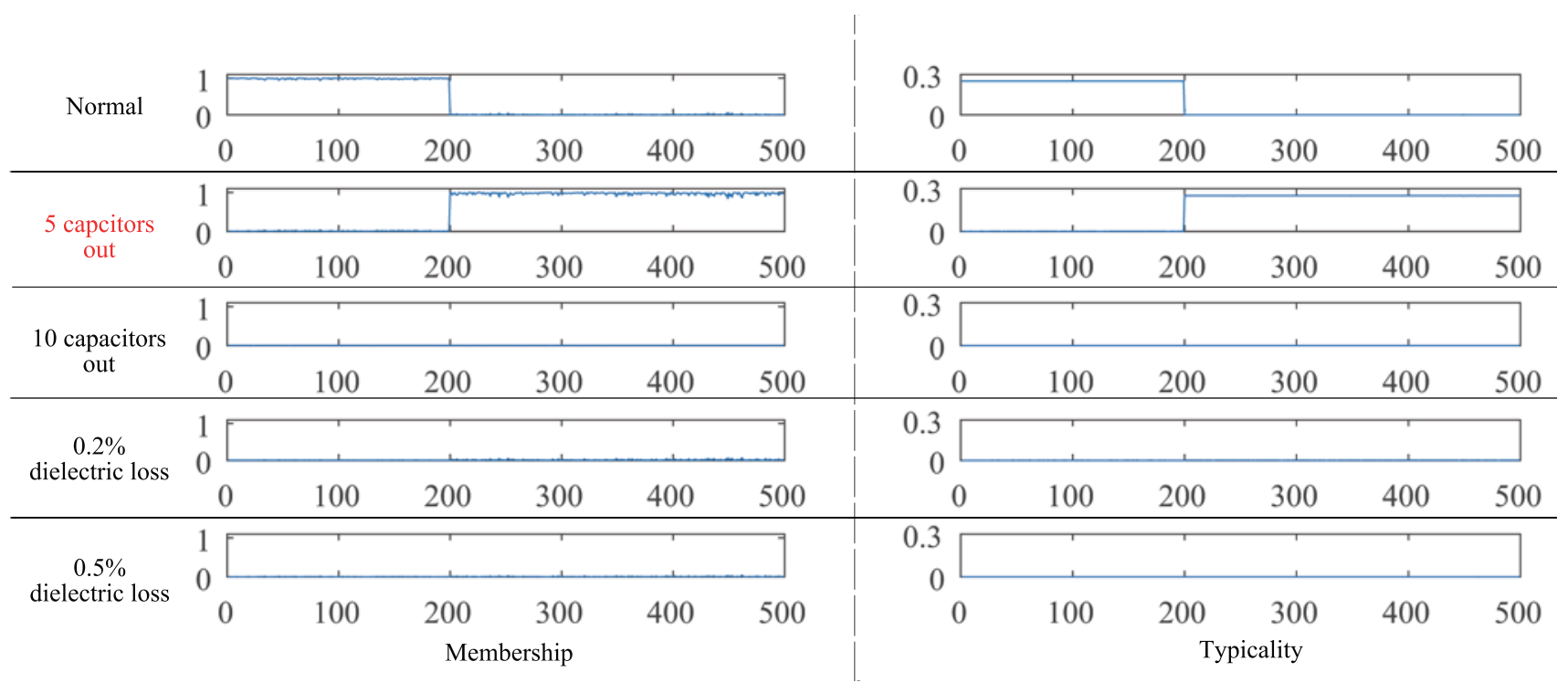




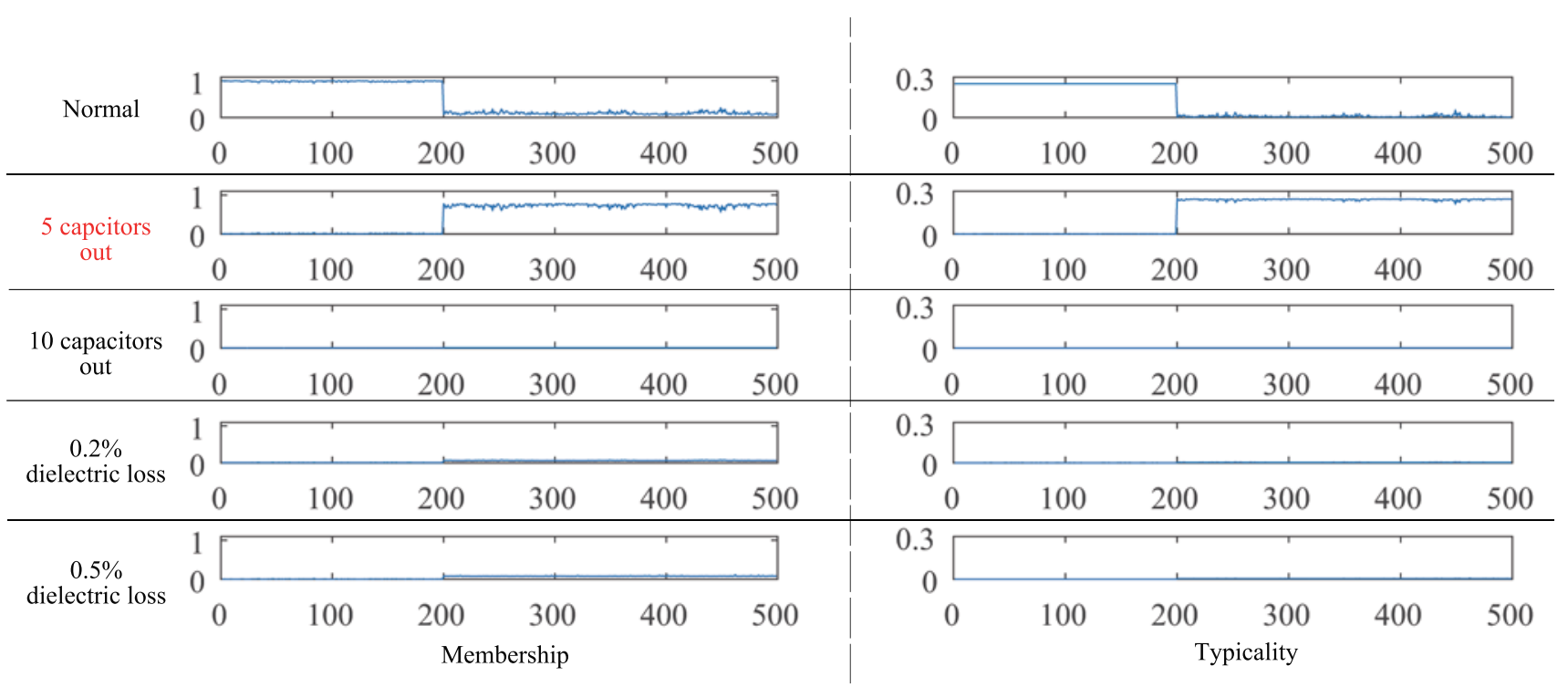




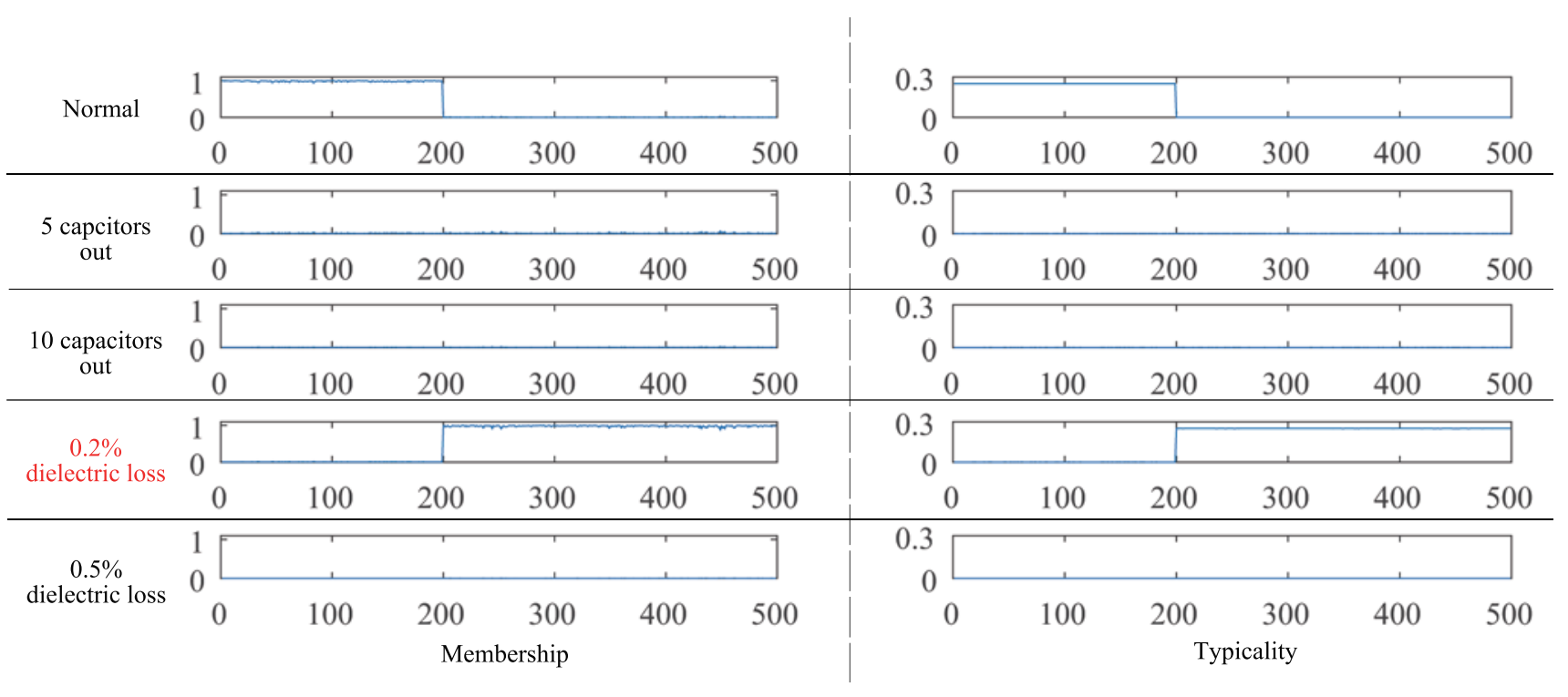




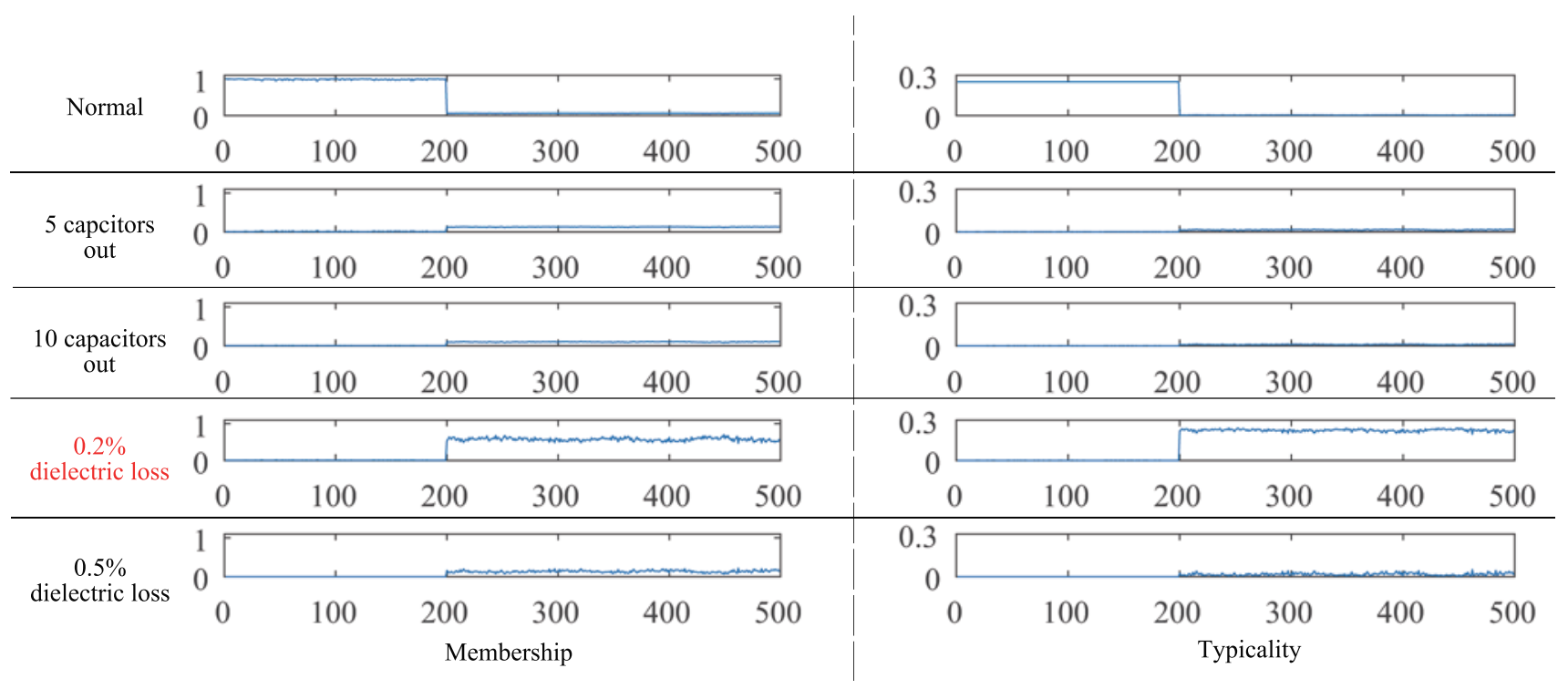




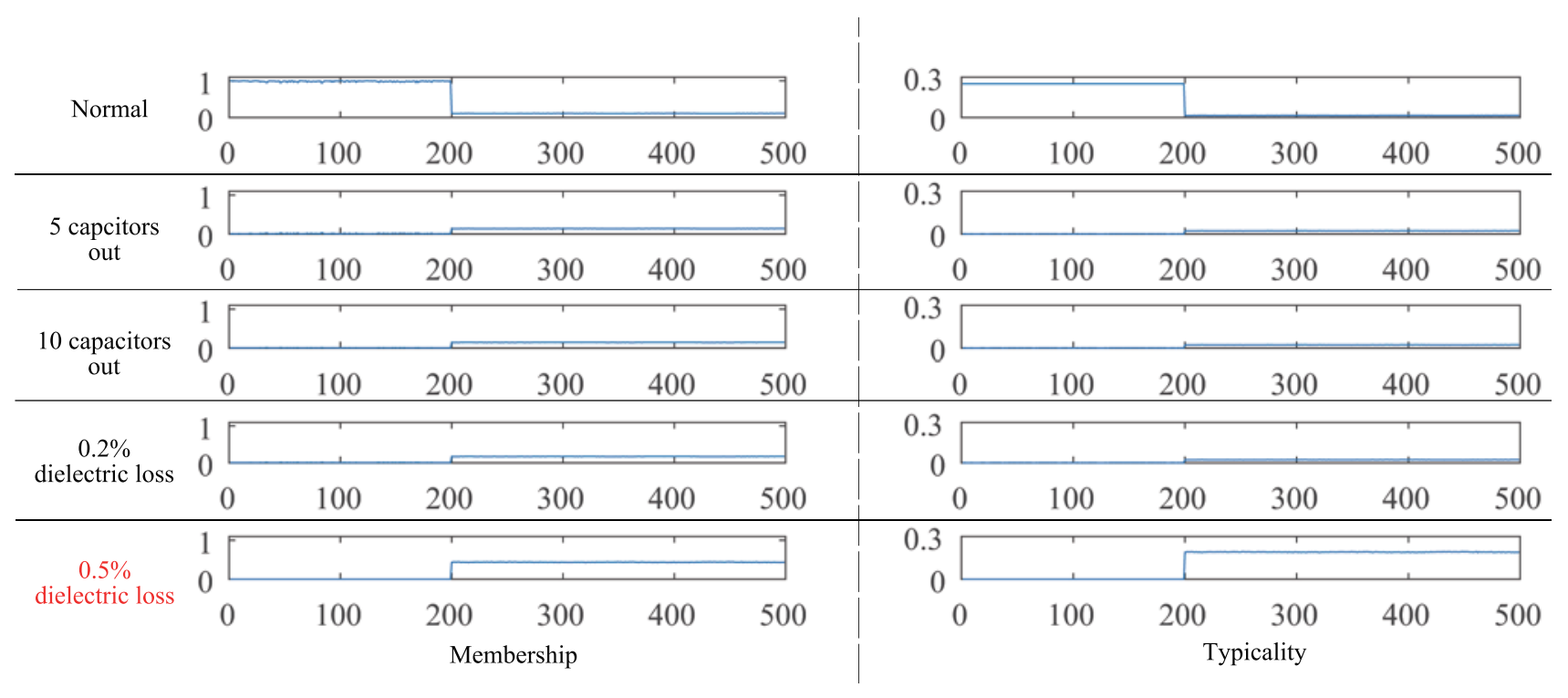




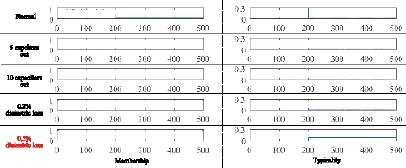

